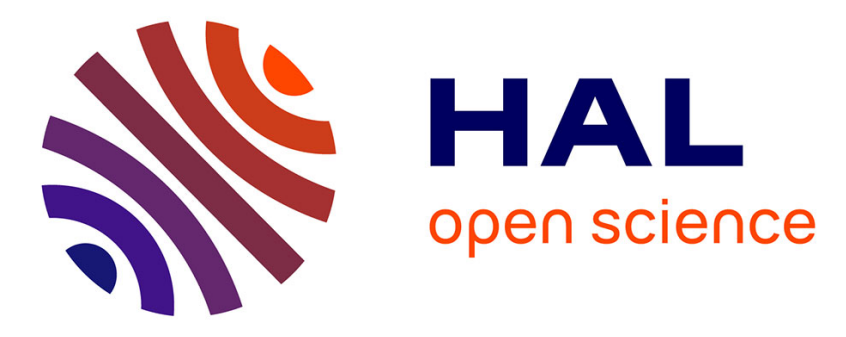

\title{
Transient perturbation growth in time-dependent mixing layers
}

Cristobal Arratia, C. P. Caulfield, Jean-Marc Chomaz

\section{To cite this version:}

Cristobal Arratia, C. P. Caulfield, Jean-Marc Chomaz. Transient perturbation growth in timedependent mixing layers. Journal of Fluid Mechanics, 2013, 717, pp.90-133. 10.1017/jfm.2012.562 . hal-00996494

\section{HAL Id: hal-00996494 \\ https://hal-polytechnique.archives-ouvertes.fr/hal-00996494}

Submitted on 3 Jun 2014

HAL is a multi-disciplinary open access archive for the deposit and dissemination of scientific research documents, whether they are published or not. The documents may come from teaching and research institutions in France or abroad, or from public or private research centers.
L'archive ouverte pluridisciplinaire HAL, est destinée au dépôt et à la diffusion de documents scientifiques de niveau recherche, publiés ou non, émanant des établissements d'enseignement et de recherche français ou étrangers, des laboratoires publics ou privés. 


\title{
Transient perturbation growth in time-dependent mixing layers
}

\author{
C. Arratia ${ }^{1, \dagger}$, C. P. Caulfield ${ }^{2,3}$ and J.-M. Chomaz ${ }^{1}$ \\ ${ }^{1}$ LadHyX, CNRS-École Polytechnique, F-91128 Palaiseau, France \\ ${ }^{2}$ BP Institute, University of Cambridge, Madingley Rise, Madingley Road, Cambridge CB3 OEZ, UK \\ ${ }^{3}$ Department of Applied Mathematics and Theoretical Physics, University of Cambridge, Centre for \\ Mathematical Sciences, Wilberforce Road, Cambridge CB3 OWA, UK
}

(Received 10 January 2012; revised 8 November 2012; accepted 13 November 2012;

first published online 1 February 2013)

We investigate numerically the transient linear growth of three-dimensional (3D) perturbations in a homogeneous time-evolving mixing layer in order to identify which perturbations are optimal in terms of their kinetic energy gain over a finite, predetermined time interval. We model the mixing layer with an initial parallel velocity distribution $\boldsymbol{U}(y)=U_{0} \tanh (y / d) \boldsymbol{e}_{x}$ with Reynolds number $R e=U_{0} d / v=1000$, where $v$ is the kinematic viscosity of the fluid. We consider a range of time intervals on both a constant 'frozen' base flow and a time-dependent two-dimensional (2D) flow associated with the growth and nonlinear saturation of two wavelengths of the most-unstable eigenmode of linear theory of the initial parallel velocity distribution, which rolls up into two classical Rayleigh instabilities commonly referred to as Kelvin-Helmholtz (KH) billows, which eventually pair to form a larger vortex. For short times, the most-amplified perturbations on the frozen tanh profile are inherently $3 \mathrm{D}$, and are most appropriately described as oblique wave 'OL' perturbations which grow through a combination of the Orr and lift-up mechanisms, while for longer times, the optimal perturbations are 2D and similar to the $\mathrm{KH}$ normal mode, with a slight enhancement of gain. For the time-evolving KH base flow, OL perturbations continue to dominate over sufficiently short time intervals. However, for longer time intervals which involve substantial evolution of the primary KH billows, two broad classes of inherently 3D linear optimal perturbation arise, associated at low wavenumbers with the well-known core-centred elliptical translative instability, and at higher wavenumbers with the braid-centred hyperbolic instability. The hyperbolic perturbation is relatively inefficient in exploiting the gain of the OL perturbations, and so only dominates the smaller wavenumber (ultimately) core-centred perturbations when the time evolution of the base flow or the start time of the optimization interval does not allow the OL perturbations much opportunity to grow. When the OL perturbations can grow, they initially grow in the braid, and then trigger an elliptical core-centred perturbation by a strong coupling with the primary KH billow. If the optimization time interval includes pairing of the primary billows, the secondary elliptical perturbations are strongly suppressed during the pairing event, due to the significant disruption of the primary billow cores during pairing.

Key words: free shear layers, instability, transition to turbulence

† Current address: LFMI, École Polytechnique Fédérale de Lausanne, CH1015 Lausanne, Switzerland. Email address for correspondence: cristobal.arratia@gmail.com 


\section{Introduction}

Gaining an understanding of the mechanisms by which initially laminar flows undergo the transition to disordered turbulent motion is one of the fundamental challenges of fluid dynamics research. A particularly important archetype flow is the so-called mixing layer, where the fluid has initially a vertical ('cross-stream'), inflectional and monotonic variation in streamwise velocity, due for example to viscous diffusion of a step-change in velocity some distance downstream of a splitter plate. Provided the flow Reynolds number $R e=U d / v$ is sufficiently large (where $U$ is some characteristic scale of the streamwise velocity which varies over a characteristic vertical, or equivalently cross-stream, distance $d$, and $v$ is the kinematic viscosity of the fluid) it is very well-known that this flow is susceptible to a strong primary instability, commonly referred to as the Kelvin-Helmholtz $(\mathrm{KH})$ instability, though in an unstratified flow where the mixing layer has a finite depth and an inflectional velocity profile, it is perhaps more appropriate to refer to the instability as a 'Rayleigh' instability (see for example Drazin \& Reid 1981 for a fuller discussion).

The primary instability manifests itself at finite amplitude as a two-dimensional (2D) train of elliptical spanwise vortices (centred on elliptical stagnation points), which roll up from the initial strip of spanwise vorticity, and are connected by 'braid' regions of high strain and depleted (spanwise) vorticity, in turn centred on hyperbolic fixed points. These primary instabilities have been observed in experiment (e.g. Brown \& Roshko 1974) and numerical simulation (Metcalfe et al. 1987) and are known to be strongly subject to subharmonic merging quasi-two-dimensional instabilities which lead to an increase in depth of the mixing layer (Winant \& Browand 1974). However, it appears that they are merely an intermediate stage in the approach of a flow to the 'mixing transition' (see Dimotakis 2005 for a review) which seems to require the development of some secondary, inherently three-dimensional (3D) instability, which upon growth to finite amplitude and interaction with the primary billow train leads to a breakdown to small-scale motions, and a marked increase in dissipation characteristic of turbulent motion. In this paper, we focus on these (experimentally observed) 3D perturbations. We consider three different situations. First, we consider transient perturbation growth on a 'frozen' inflectional shear layer, where the base flow does not vary with time so that we are able to assess the extent to which variation in the base flow plays a role in the properties of the optimal perturbations. Secondly, we deliberately filter out the complicating effect of subharmonic merging instabilities by restricting the time-dependent flow to a streamwise extent equivalent to the wavelength of the most unstable KH 'billow'. For this situation, we only consider sufficiently short time optimization intervals so that two billows would not be expected to interact strongly. Armed with this understanding, finally we then consider the dynamics of two billows, which we allow to pair, to investigate the secondary perturbations which can grow on such a strongly evolving flow. We are particularly interested in whether these perturbations can be related to the perturbations which grow locally on a single billow.

There have been two main candidates proposed for such 'local' secondary instabilities which allow the transition to turbulence. Since the primary KH billows are elliptical (and centred on elliptical stagnation points of the flow) and are affected by the strain field associated with their neighbours, it has often been hypothesized that they may be subject to a relatively low-wavenumber, inherently 3D 'elliptical instability' of a strained elliptical vortex (see Pierrehumbert \& Widnall 1982; Bayly 1986; Waleffe 1990; Kerswell 2002 for a comprehensive review). Although numerical simulations do show evidence of core-centred perturbations, the most noticeable $3 \mathrm{D}$ structures in both experiment and numerical simulations are relatively 
higher wavenumber 'rib vortices' (see Hussain 1983 for an early review), i.e. thin essentially streamwise-aligned braid-centred vortices that wrap around the primary billow cores. This apparent mismatch in the initial growth location and the finiteamplitude manifestation for these rib vortices was initially a major point of concern for theoreticians.

Pierrehumbert \& Widnall (1982) had identified a core-centred instability (which they referred to as the 'translative instability' due to the fact that this instability is characterized by a spanwise-periodic displacement of the vortical core) on a periodic row of Stuart vortices and speculated as to their relevance for the streamwise vortices observed in mixing layers. The fact that the translative instability is localized in the vortex cores led Corcos \& Lin (1984) to speculate about a different mechanism: '... it is likely that the strong streamwise vorticity that appears and persists in the central part of the braids, and which is responsible for the streamwise streaks...is caused early on by the original (3D) shear instability rather than by the translative instability, and thereafter lives a fossil life'. That the dynamics of the initial stages of the flow could play a role on the later development of the mixing layer is stressed by Corcos \& Lin (1984): 'Either nonlinear interactions of waves of neighbouring spanwise wavenumber (particularly difficult to study numerically over a finite domain) or the competitive advantage given by particular initial conditions may lead to a selective mechanism'. A precise assessment of these conjectures was difficult at that time. Subsequent research on mechanisms causing the observed 3D features of shear layers has focused mainly on secondary instabilities present on the fully developed KH billows; this program succeeded in finding various unstable modes and instability mechanisms.

Of particular significance was the secondary stability analysis of Klaassen \& Peltier (1991), which made use of a methodology developed earlier by the same authors (Klaassen \& Peltier 1985) involving the (strong) assumption that the primary billow is completely frozen in time. Klaassen \& Peltier (1991) showed that the primary billow flow, consisting of a snapshot of a 2D direct numerical simulation (DNS), was also susceptible to another secondary 3D 'hyperbolic' instability, at substantially smaller wavelength than the elliptical instability and centred on the braid region between neighbouring billow cores. Another distinguishing characteristic between the two instabilities is that the elliptical instability, associated as it is with periodic deflections of the primary billow core, is associated with substantial spanwise perturbation vorticity, while the hyperbolic instability is associated with substantial vertical (crossstream) and streamwise perturbation vorticity between neighbouring primary billow cores.

Numerical simulations (for example Rogers \& Moser 1992; Caulfield \& Peltier 2000, who also confirmed the analysis of Klaassen \& Peltier 1991 at higher resolution) suggest strongly that both elliptical and hyperbolic instabilities can occur within the evolving flow. At finite amplitude, the rib streamwise-aligned vortices appear to be braid-centred as is the case for the linear modes of the hyperbolic instability. Therefore, there is a strong suggestion that the hyperbolic instability plays the key role in transition. Nevertheless, since the primary billows are indeed susceptible to 'elliptical' or 'translative' instabilities as well, there is every chance that the complicated interactions conjectured by Corcos \& Lin (1984) may well occur. Indeed, numerical simulations show that perturbations in the braid lead to streamwise-aligned rib vortices and substantial perturbation of the billow core, and in some way the combination leads to transition, leading Rogers \& Moser (1992) to argue that: 'While it may be useful, in attempting to understand the translative instability, to distinguish 
between instability mechanisms that are localized to the roller cores or the braid region, the results discussed above make it clear that perturbations in the core and braid grow together. Therefore, in interpreting results of experiments or simulations, the $3 D$ instability should be considered to be a global instability of the entire flow'.

However, the central 'frozen-in-time' assumption of the analysis presented by Klaassen \& Peltier (1991) is not completely supported by evidence from numerical simulations. If seeded with a small-amplitude initial perturbation, the primary billow instability does indeed saturate at finite amplitude, after a period of close to exponential growth. Even in the absence of merger, the primary billow is by no means steady, but undergoes quasi-periodic oscillations, with continuous exchange of energy occurring between the base flow and the perturbation. This particular issue was addressed in detail by the analysis of Smyth \& Peltier (1994) who presented further arguments concerning the conditions under which it might be reasonable to assume that the base flow is frozen in time. The key issue they identified is whether there is substantial time scale separation between the evolving base flow and the time scale of the growth of the secondary instabilities. When the base flow was evolving, they developed a new innovative technique using what they referred to as a time-averaged stability matrix or TASM, which 'averaged the base fields over the time interval', and then used this averaged base flow as the generalization of the frozen base flow in their analysis. They argued that this approach leads to an improved algorithm for determining the properties of secondary perturbation growth on a time-dependent flow, and furthermore specifically for a (non-rotating) mixing layer, they argued that the time-dependence of the base flow was especially significant only when the optimization time interval straddled the pairing of the primary billows.

However, their approach introduces an approximation through averaging of the actual time-dependent flow, and so it is undoubtedly interesting to investigate whether this approximation changes the perturbations which have the largest growth, and so are expected to develop in a realistic flow. Indeed, as noted by Potylitsin \& Peltier (1998) the TASM method is flawed, not least because the averaging inevitably smooths out spatial and temporal variation in the base flow, which may play a critical role in the growth of secondary perturbations. We return to a discussion of this issue in more detail in $\$ \S 5.4$ and 6. Furthermore, from such frozen-in-time or averaged analyses it is natural to think of elliptical and hyperbolic instabilities as instabilities of the saturated primary billow, or some averaged form of the pairing billows, or even the ultimate paired subharmonic elliptical vortex, with the essentially time-dependent developed vortex or vortices acting as a catalyst for the onset of the secondary instabilities. But in reality the primary billow takes a certain amount of time to roll up to its saturated state, and so a natural open question is the extent to which significant perturbations can grow on the time-evolving base flow state, as it is at least possible that these perturbations might be growing right from when the flow is organized as a simple, essentially parallel inflectional shear flow. This roll-up phase is another phase of the flow's evolution, distinct from the later-time pairing phase of the primary billows, when the inherent time-dependence of the flow may be, at least in principle, important in the selection of the appearing 3D flow features. Whereas the selection process may clearly depend on the structure of the specific initial condition, in particular the perturbation from the base flow, a general characterization can be provided by a computation of the optimally growing perturbations. The extent to which an initial perturbation projects onto perturbations which can grow is of course important as this naturally determines the initial amplitude of the forcing of each potentially growing perturbation. However, it is also important to remember that the (in general 
time-dependent) growth characteristics of each such perturbation are also central to determining the finite-amplitude character of the evolving flow at some finite horizon time.

Naturally, considering such problems of the development of perturbations on a timeevolving underlying flow is further complicated by the inherent non-normality of the linearized Navier-Stokes operator. Because of this, it is well-known that the energy of the perturbations can grow transiently (i.e. over a finite time interval) on a stationary base flow even in the absence of a primary 'normal-mode' instability, or alternatively at a rate transiently larger than the underlying normal-mode growth rate, and the development of non-modal stability theory (see Schmid 2007 for a review) allows the investigation of the role of initial conditions over finite time intervals in a time-varying base flow state in a systematic way. Indeed, there is evidence from simple models of the braid region, as presented by Caulfield \& Kerswell (2000), that the hyperbolic instability is particularly suited to transient growth, and recently, Ortiz \& Chomaz (2011) have identified a new possible growth mechanism for braid-like regions, which they relate to the 'anti-lift-up' mechanism previously described by Antkowiak \& Brancher (2007). Smyth \& Peltier (1994) noted that the modes which they identified were non-orthogonal, and so they were able, for either the frozen or time-averaged base flow state to identify the optimal transient perturbation over a given time horizon. However, what the optimal perturbation on an evolving mixing layer flow is remains an open and interesting question particularly over varying time horizons. Although if there is a truly unstable class of modes (with positive exponential growth rates) the mode with the largest growth rate is expected to dominate over sufficiently long time horizons, it is not clear a priori whether transient effects will lead to substantially enhanced gain, or indeed when the cross-over to the most unstable (true) mode will occur.

From a physical viewpoint, there are two natural mechanisms which have been identified as causing (energetic) transient growth of perturbations in shear flows, known as the Orr mechanism (as originally presented by Orr 1907) and the lift-up mechanism (see Ellingsen \& Palm 1975; Landahl 1980). The Orr mechanism involves counter-rotating vortices which are parallel to the base-flow (and hence spanwise) vorticity being tilted into and against the mean shear and hence amplified by the base shear via the working of the Reynolds stress. This mechanism is efficient at relatively high streamwise wavenumbers (i.e. small scales) on short time scales as demonstrated by Butler \& Farrell (1992). On the other hand, in the lift-up mechanism, streamwise vortices interact with the basic shear to generate streamwise perturbation velocity. As shown by Butler \& Farrell (1992), this mechanism operates at somewhat smaller wavenumbers, and on somewhat larger time scales. Naturally, in an intermediate range of either wavenumber or time interval, combinations or 'mixtures' of these two growth mechanisms can occur, as discussed for example by Guegan, Huerre \& Schmid (2007), and these mechanisms at least have the potential to force transient perturbations which grow more rapidly than classic, linearly unstable modes.

Much of the focus of research into 'optimal' transient growth (i.e. the identification of perturbations which have the largest relative growth in their perturbation kinetic energy or gain over some finite time interval) has been on flows such as plane Couette flow or pipe flow where there is no linear instability, or channel flow where the linear instability onsets at a much higher Reynolds number than transition is observed to occur in experiment and simulations. In such circumstances, the transiently growing perturbations will clearly dominate the flow evolution. On the other hand, if there are unstable normal modes, it is not immediately obvious what role, if any, is played 
by transient perturbations (utilizing the Orr mechanism, the lift-up mechanism, or indeed some mixture of the two) in the flow evolution. The conventional picture (consistent with experimental and numerical evidence) is that the flow will be ultimately dominated by the most-unstable (eigen) mode of linear theory. However, it is not clear how long this process takes, and it is at least conceivable that an appropriately chosen initial perturbation may grow very rapidly, at least over a short time. Such a transient perturbation, allowed to develop to finite amplitude, and thus to modify the base flow in a non-trivial manner, may actually preclude the development of the primary $\mathrm{KH}$ billow, leading potentially to a kind of 'bypass' transition with no primary instability development, as can be seen for example in the (unstratified) DNS of Basak \& Sarkar (2006).

In summary, there are therefore three key open questions of interest. First, are the previously identified elliptical and/or hyperbolic instabilities 'optimal' perturbations of an inflectional shear layer in any sense? Secondly, what is the role of the time-dependence of the evolving billow in the development of optimal growing perturbations? In particular, do elliptical and hyperbolic instabilities rely fundamentally on the primary billow being fully saturated, or can they grow as the primary billows roll up and potentially pair? And thirdly, how relevant are the simple idealized pictures of modal instability and physical growth mechanisms to the actual development of perturbations within an inflectional shear layer flow?

These questions will be addressed in this paper using the tools of non-modal stability analysis (as discussed in detail in the review of Schmid 2007). In particular a linearized time-stepping Navier-Stokes equation solver and its adjoint will be 'looped' and hence iterated multiple times to identify the properties of the optimal linear perturbation (in the sense that the relative gain of the kinetic energy of the perturbation is maximized over some time interval). This power iteration looping method is very well suited to the problem at hand, as this method can straightforwardly embed the properties of a time-evolving base state in the equation solvers (both direct and adjoint) which are used. Indeed, with an evolving base flow, there is an interesting mathematical subtlety to do with the fact that not only is the length of the time interval over which optimization of gain occurs important, but also the chosen start time (in the evolution of the base flow state) is significant to the secondary perturbations. A particular focus will be to compare our results to those in the literature, particularly where frozen-in-time or the related TASM approaches have been used.

This paper is organized as follows. After briefly introducing the (largely now conventional) mathematical formulation and algorithm in $\S 2$, focusing on the implications of using a time-evolving base flow state, we first consider transient perturbation growth (over a wide range of optimizing time intervals) on an initially hyperbolic-tangent base state flow that is frozen in time by the application of a body force. In $\S 3$, we focus in particular on identifying the time interval over which the conventional $\mathrm{KH}$ instability mode is not the optimal response. Having considered transient perturbation growth on the steady hyperbolic-tangent base flow, we generate a time-evolving 2D single $\mathrm{KH}$ billow at sufficiently high Reynolds number to grow to a non-trivial finite amplitude. In $\S 4$ we then consider transient perturbation growth on this time-evolving, non-parallel billow base flow over a range of time intervals. We classify and characterize the predicted optimal perturbations both in terms of the spatial distribution of energy, and also the relative size of the components of the enstrophy. This compartmentalization of the enstrophy is very useful to interpret the properties of the (in general) 3D perturbations, and to identify any possible connection 
to the previously predicted elliptical and hyperbolic instabilities, as well as the crucial physical growth mechanisms. In $\S 5$ we then also consider the importance of the chosen start time of the optimization time interval relative to the time-evolution of the primary $\mathrm{KH}$ billow on the optimal perturbation, discussing the possible significance of the anti-lift-up mechanism described in Ortiz \& Chomaz (2011) for initial perturbation growth in the braid. We also compare our results to those of Smyth \& Peltier (1994), considering the influence of the flow's Reynolds number, and the significance of either the frozen-in-time or the time-averaged stability matrix (TASM) approximations to the actual time-varying base flow. In $\S 6$, we then consider the case where the primary billows are allowed to pair, and so the base flow is rapidly varying, thus violating any assumption concerning a separation of time scales between the evolution of perturbations and the base flow. Finally, in $\$ 7$ we draw our conclusions, and present suggestions for future work, particularly considering the possible use of full nonlinear direct numerical simulations to investigate the finite-amplitude evolution of the identified optimal perturbations.

\section{Mathematical formulation}

We consider the linear evolution of perturbations $\boldsymbol{u}_{p}$ to a base flow $\boldsymbol{U}(t)$ under the incompressible Navier-Stokes equations. The base flow $\boldsymbol{U}=(U(x, y, t), V(x, y, t), 0)^{\mathrm{T}}$ is restricted to (at most) two dimensions, while the perturbation $\boldsymbol{u}_{p}$ is allowed to evolve in three dimensions. We choose a coordinate system so that $x$ is in the streamwise direction, $y$ is in the vertical (or equivalently cross-stream) direction, and $z$ is in the spanwise direction. Scaling the flow variables with the characteristic velocity scale $U$ and the shear layer length scale $d$ (mentioned in the introduction) to define the Reynolds number, the non-dimensional linearized Navier-Stokes equations are thus

$$
\begin{aligned}
\frac{\partial \boldsymbol{u}_{p}}{\partial t}+(\boldsymbol{U}(t) \cdot \nabla) \boldsymbol{u}_{p} & =-\nabla p_{p}-\left(\boldsymbol{u}_{p} \cdot \nabla\right) \boldsymbol{U}(t)+R e^{-1} \nabla^{2} \boldsymbol{u}_{p}, \\
\nabla \cdot \boldsymbol{u}_{p} & =0 .
\end{aligned}
$$

The boundary conditions are periodic in $x$ and $z$, with $\boldsymbol{u}_{p}$ and $\nabla p_{p}$ tending to zero when $|y| \rightarrow \infty$.

Formally, the calculations of solutions to the governing equations (2.1) may be thought of as a propagator of the (perturbation) velocity field from some initial time $t_{i}$ to some final time $t_{f}$, i.e.

$$
\boldsymbol{u}_{p}\left(t_{f}\right)=\Phi_{\lambda}\left(t_{f}, t_{i}\right) \boldsymbol{u}_{p}\left(t_{i}\right)
$$

where the sub-index $\lambda$ represents the parameters, the Reynolds number and the geometry (and perhaps discretization in practice) of the flow domain under consideration. We are thus interested in the optimization of the gain $\mathscr{G}\left(\lambda, T, T_{0}\right)$ defined as

$$
\begin{aligned}
\mathscr{G}\left(\lambda, T, T_{0}\right) \equiv \frac{E(\lambda, T)}{E\left(\lambda, T_{0}\right)} & =\frac{\left\langle\boldsymbol{u}_{p}(T), \boldsymbol{u}_{p}(T)\right\rangle}{\left\langle\boldsymbol{u}_{p}\left(T_{0}\right), \boldsymbol{u}_{p}\left(T_{0}\right)\right\rangle} \\
& =\frac{\left\langle\Phi_{\lambda}\left(T, T_{0}\right) \boldsymbol{u}_{p}\left(T_{0}\right), \Phi_{\lambda}\left(T, T_{0}\right) \boldsymbol{u}_{p}\left(T_{0}\right)\right\rangle}{\left\langle\boldsymbol{u}_{p}\left(T_{0}\right), \boldsymbol{u}_{p}\left(T_{0}\right)\right\rangle} \\
& =\frac{\left\langle\boldsymbol{u}_{p}\left(T_{0}\right), \Phi_{\lambda}^{\dagger}\left(T, T_{0}\right) \Phi_{\lambda}\left(T, T_{0}\right) \boldsymbol{u}_{p}\left(T_{0}\right)\right\rangle}{\left\langle\boldsymbol{u}_{p}\left(T_{0}\right), \boldsymbol{u}_{p}\left(T_{0}\right)\right\rangle},
\end{aligned}
$$


where angled brackets denote the conventional inner product yielding the energy $E$, and the superscript $\dagger$ denotes the adjoint such that

$$
\left\langle\boldsymbol{u}_{d}, \Phi_{\lambda} \boldsymbol{u}_{p}\right\rangle=\left\langle\Phi_{\lambda}^{\dagger} \boldsymbol{u}_{d}, \boldsymbol{u}_{p}\right\rangle
$$

for all $\boldsymbol{u}_{d}$ and $\boldsymbol{u}_{p}$. Without loss of generality, we normalize the initial condition so that $E\left(\lambda, T_{0}\right)=1$.

It can be easily seen that the adjoint of the propagator $\Phi_{\lambda}^{\dagger}\left(T, T_{0}\right)$ corresponds to the propagator of the adjoint equations from $T$ to $T_{0}$. (For time-dependent equations, this can be directly shown by taking the adjoint of the propagator as given in equation (4) of Farrell \& Ioannou 1996.) It is straightforward to establish (see for example Hill 1995; Donnadieu et al. 2009) that the adjoint equations of (2.1) are

$$
\begin{aligned}
\frac{\partial \boldsymbol{u}_{d}}{\partial \tau} & =\boldsymbol{\Omega}(-\tau) \times \boldsymbol{u}_{d}-\nabla \times\left(\boldsymbol{U}(-\tau) \times \boldsymbol{u}_{d}\right)-\nabla p_{d}+R e^{-1} \nabla^{2} \boldsymbol{u}_{d}, \\
\nabla \cdot \boldsymbol{u}_{d} & =0 .
\end{aligned}
$$

In these equations $\tau=-t, \Omega=\nabla \times \boldsymbol{U}, \boldsymbol{u}_{d}$ is the adjoint velocity variable, $p_{d}$ is the equivalent 'pressure' adjoint variable enforcing incompressibility, the boundary conditions are the same as for (2.1), and the initial condition according to $(2.3 c)$ is $\boldsymbol{u}_{d}(T)=\Phi_{\lambda}\left(T, T_{0}\right) \boldsymbol{u}_{p}\left(T_{0}\right)$. As is commonly understood, due to the integration by parts that is implicit in the definition of the adjoint, the relative signs of the time derivative and the Laplacian are different for the direct and the adjoint Navier-Stokes equations, implying that the adjoint equations are well-posed when integrated backwards in time. It is also clear that even if the propagator $\Phi_{\lambda}$ is not self-adjoint, the combination $\Phi_{\lambda}^{\dagger} \Phi_{\lambda}$ is self-adjoint. The optimal perturbation is that which attains the maximum gain

$$
\mathscr{G}_{\max }\left(\lambda, T, T_{0}\right)=\max _{\boldsymbol{u}_{p}\left(T_{0}\right)}\left\{\mathscr{G}\left(\lambda, T, T_{0}\right)\right\},
$$

where the maximization is over all choices of initial conditions $\boldsymbol{u}_{p}\left(T_{0}\right)$. Since the operator $\Phi_{\lambda}^{\dagger} \Phi_{\lambda}$ is self-adjoint this maximum is given by its leading eigenmode. Throughout this paper we will set the central flow parameter $R e=1000$ (except in $\S 5.4$, where we study the effect of variations in $R e$ ), which is sufficiently large to ensure that the primary instability rolls up into a finite-amplitude, energetic billow.

These adjoint equations can be solved using a straightforward modification of a pseudo-spectral DNS code with an Adams-Bashforth time scheme (as previously described and utilized by Donnadieu et al. 2009). In particular, the central point arising from the integration by parts that the Laplacian operator has an opposite sign (and hence that the adjoint propagator is well-posed when integrating backward in time) is very simple to implement computationally. The iterative optimization algorithm essentially relies on power iteration (see Schmid 2007 for more details). A guess for the initial conditions $\boldsymbol{u}_{0}$ is integrated forwards in time from $t=T_{0}$ to the target time $t=T$ using the (forward) propagator $\Phi_{\lambda}$. The final state $\boldsymbol{u}_{T}$ is then used as the initial conditions for the adjoint propagator $\Phi_{\lambda}^{\dagger}$, which is then integrated backward in time from $T$ to $T_{0}$. This final state (after appropriate rescaling, see for example (3.5)) is then used as the initial condition for the next loop of this iteration. Multiple iterations of this entire loop will naturally lead to the solution being dominated by the eigenfunction of the (combined) operator $\Phi_{\lambda}^{\dagger} \Phi_{\lambda}$ associated with the eigenvalue with the largest real part. This leading eigenmode of course corresponds (up to scaling) to the initial perturbation which has the largest gain over the target time interval. 


\section{Transient response of a frozen parallel hyperbolic-tangent shear flow}

\subsection{Base flow and decomposition of perturbations}

As discussed in the introduction, the first problem we consider is the transient growth of infinitesimally small perturbations on a steady parallel inflectional shear flow. We consider a tanh profile

$$
\boldsymbol{U}(y)=\tanh (y) \boldsymbol{e}_{x},
$$

as an appropriate choice for this parallel base flow. Due to the fact that the flow we consider has finite diffusion (with $R e=1000$ ), over time such a profile will spread vertically (or equivalently cross-stream). Therefore, for the base flow (3.1) to be a solution to the governing equations, we formally add a body force,

$$
\boldsymbol{F}=-R^{-1} \tanh (y)^{\prime \prime} \boldsymbol{e}_{x},
$$

to the full Navier-Stokes equations (where primes denote $y$-derivatives) so that $\boldsymbol{U}(y)=\tanh (y) \boldsymbol{e}_{x}$ is actually a solution. This is done in classical stability studies (Drazin \& Reid 1981) and yields (2.1a) without a source term from base flow diffusion. Therefore, the perturbations are evolving in a flow with finite $R e$, but the base (parallel) flow $\boldsymbol{U}(y)$ defined by (3.1) is frozen.

Because the governing equations (2.1) with the base flow (3.1) have coefficients with no dependence on $x$ and $z$, we can spectrally decompose the perturbations as

$$
\left[\boldsymbol{u}_{p}, p_{p}\right](x, y, z, t)=\operatorname{Re}\left([\boldsymbol{u}, p](y, t) \exp \left(\mathrm{i}\left(k_{x} x+k_{z} z\right)\right)\right) .
$$

We can then compute the $y$-dependent eigenfunctions $[\boldsymbol{u}, p]$ independently for each $\left(k_{x}, k_{z}\right)$. This computation can be implemented efficiently within a 3D linearized code, as there is no interaction between modes with different $\left(k_{x}, k_{z}\right)$ combinations. We can thus identify the energy of each of the perturbations as

$$
E\left(k_{x}, k_{z}, t\right)=\int_{y_{\min }}^{y_{\max }} \boldsymbol{u}^{*} \cdot \boldsymbol{u} \mathrm{d} y,
$$

where ${ }^{*}$ denotes the complex conjugate, and $y_{\min }=-15$ and $y_{\max }=15$ are the minimum and maximum vertical extents of the flow domain respectively. It is also important to note that the energy is normalized so that the total energy on each of the modes is one initially, i.e.

$$
E\left(k_{x}, k_{z}, T_{0}\right)=1,
$$

so that consecutive iterations converge to the optimal perturbation for all the computed $\left(k_{x}, k_{z}\right)$, avoiding potential computational problems if the less-amplified modes were allowed to have significantly smaller amplitude than the most amplified ones. A convenient way to characterize the various perturbations is by using the mean optimal growth rate

$$
\sigma_{m}\left(k_{x}, k_{z}, T\right)=\frac{\ln \left[\mathscr{G}_{\max }\left(k_{x}, k_{z}, T\right)\right]}{2 T},
$$

where the optimization is across all possible $y$-dependent eigenfunctions of the gain from $T_{0}=0$ to the target time $T$.

As the optimization time increases, unsurprisingly the maximum optimal mean growth rate (further maximized over all choices of $k_{x}$ and $k_{z}$ ) approaches from above the maximum growth rate of the classic (modal) $\mathrm{KH}$ instability, that is, $\max \left(\sigma_{m}\right) \rightarrow$ $\max \left(\sigma_{k h}\right)$ as $T \rightarrow \infty$, with the maximizing streamwise wavenumber $k_{x}^{\max } \rightarrow k_{k h}$ 
(a)

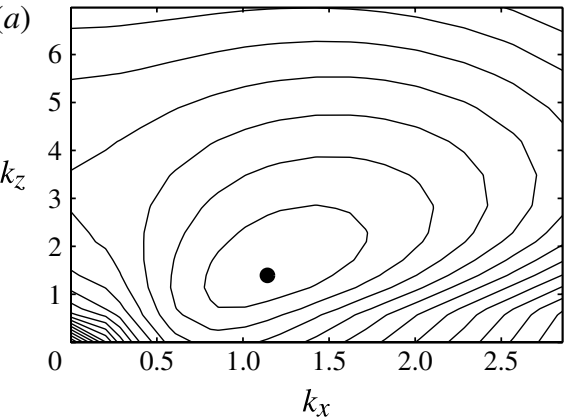

$k_{x}$ (b)

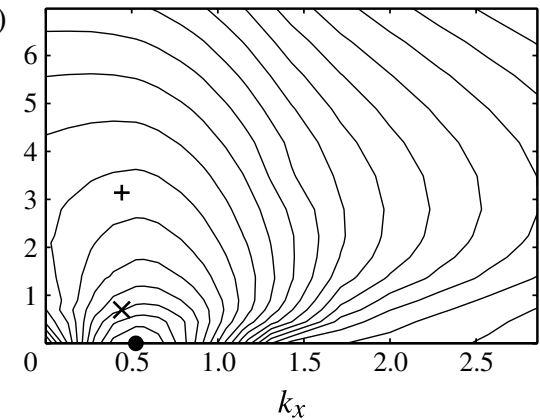

(c)

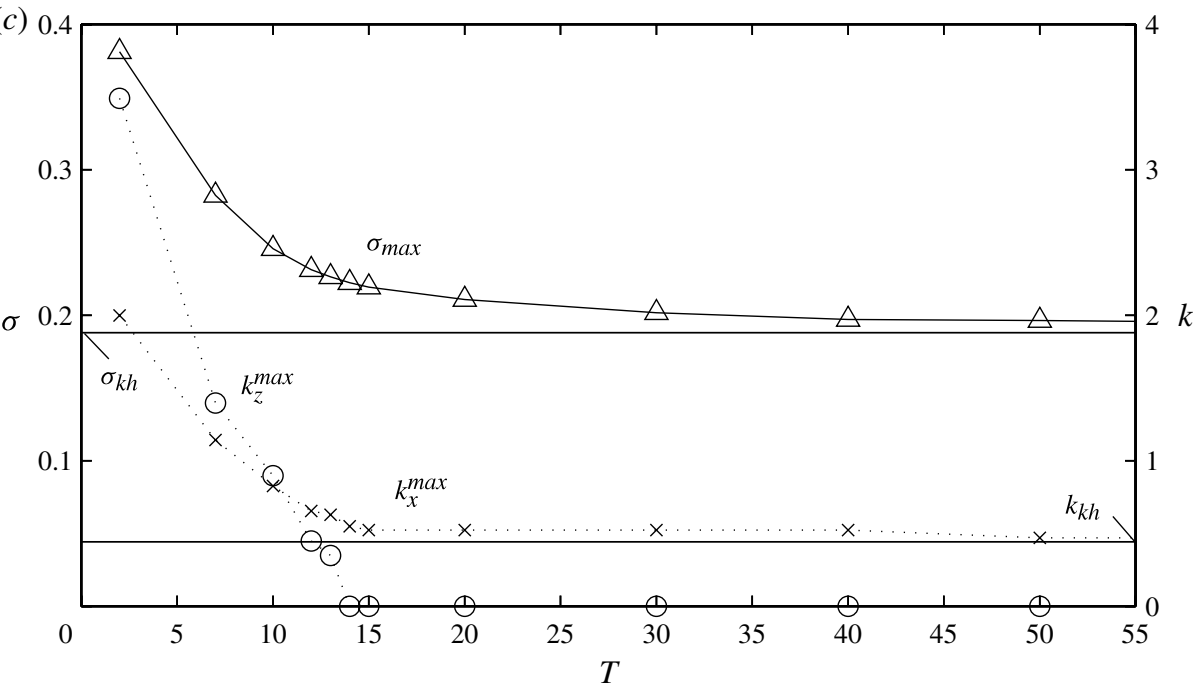

FIgURE 1. $(a, b)$ Contours of the optimal mean growth rate $\sigma_{m}$ as defined in (3.6) for optimization times $T=7(a)$ and $T=20(b)$. In both figures the contour levels are set in steps of 0.011 , decreasing from 0.275 for $T=7(a)$ and from 0.209 for $T=20(b)$. Each global maximum is indicated by a black dot $(0)$ and two characteristic 'OL-type' optimal perturbations, 'OLE' and 'OLH' as discussed in the text, are represented in $(b)$ by the $\times$ $(\mathrm{OLE})$ and the $+(\mathrm{OLH})$ symbols. $(c) \Delta, \sigma_{\max } ; \bigcirc, k_{z}^{\max }$ and $\times, k_{x}^{\max }$, i.e. maximum over $\left(k_{x}, k_{z}\right)$ of $\sigma_{m}(T)$ and associated $k_{z}$ and $k_{x}$. The upper horizontal line (associated with the left-hand vertical axis) indicates the maximum growth rate of the $\mathrm{KH}$ instability that occurs for streamwise wavenumber $k_{k h}=0.4425$ (indicated by the lower horizontal line, associated with the right-hand vertical axis) and $k_{z}=0$.

approaching that of the most unstable $\mathrm{KH}$ modal instability and $k_{z} \rightarrow 0$. A more detailed quantitative description of this behaviour is shown in figure 1.

\subsection{Optimal perturbations: OL-type and K-type}

Figure $1(a, b)$ shows the optimal mean growth rates $\sigma_{m}\left(k_{x}, k_{z}\right)$ for $T=7$ (figure $1 a$ ) and $T=20$ (figure $1 b$ ). For $T=7$, it is clear that the most amplified optimal perturbation is inherently three-dimensional (3D, i.e. with $\left.k_{z} \neq 0\right)$. The largest $\sigma_{m}$ computed is $\sigma_{\max }=0.2824$ at $\left(k_{x}^{\max }, k_{z}^{\max }\right)=(1.142,1.396)$ and is marked with a black dot in figure 1(a). Conversely, for $T=20$, the most amplified optimal perturbation is two-dimensional (2D, i.e. with $\left.k_{z}=0\right)$, and the largest $\sigma_{m}$ computed is $\sigma_{\max }=0.2109$ at $\left(k_{x}^{\max }, k_{z}^{\max }\right)=(0.5236,0)$ and marked with a black dot in figure $1(b)$. This particular 
combination of wavenumbers is in the region of parameter space that is known to be susceptible to the $\mathrm{KH}$ instability. Figure $1(c)$ shows, for different $T$, the largest computed optimal mean growth rates $\sigma_{\max }$ (left axis) and their corresponding wavenumbers $\left(k_{x}^{\max }, k_{z}^{\max }\right)$ (right axis). The horizontal lines of figure 1(c) correspond to $\sigma_{k h}=0.1881$, the growth rate of the most unstable mode of the $\mathrm{KH}$ instability at this $R e$ (upper horizontal line), and to $k_{k h}=0.4425$, the streamwise wavenumber corresponding to the most unstable mode. As $T$ increases, $\sigma_{\max }, k_{x}^{\max }$ and $k_{z}^{\max }$ decrease and approach the values corresponding to the most unstable $\mathrm{KH}$ mode, $\sigma_{k h}, k_{k h}$ and 0 , respectively. The most amplified perturbations are inherently 3D for $T \leqslant 13$, but then become $2 \mathrm{D}$ for $T \gtrsim 14$. Thus, we can distinguish between two qualitatively different types of strongly amplified optimal perturbations, which dominate depending on the particular target time chosen. We find that 3D perturbations dominate for short $T$, while 2D perturbations (clearly related to the KH normal-mode instability) dominate for larger $T$, with the cross-over occurring for this value of $R e$ around $T=15$.

As already discussed in the Introduction, the large amplification of 3D oblique wave perturbations for $T \sim 10$ is a universal feature of plane shear flow, as discussed in detail by Farrell \& Ioannou (1993b). Both the Orr (Orr 1907) and the liftup (Ellingsen \& Palm 1975) mechanisms have an effect on the evolution of 3D perturbations in plane shear flows. As discussed in detail by Farrell \& Ioannou (1993a), the large amplification for such 3D perturbations is due to a synergy between the two mechanisms; according to their description, the increased vertical (crossstream) perturbation velocity $v$ produced by the Orr mechanism excites $u$ through the lift-up mechanism. Therefore, we choose to refer to these early-time interval 3D perturbations as being of OL-type, as they may be thought of as utilizing both Orr and lift-up mechanisms. In this simplified picture (an alternative description in terms of the in-plane divergence and vorticity has been provided recently by Vitoshkin et al. 2012), the relative contribution of the Orr and lift-up mechanisms depends on the orientation of the oblique waves, going from pure Orr for $k_{z}=0$ (and perhaps some $\mathrm{KH}-$ like behaviour if in the $\mathrm{KH}$ unstable region) to pure lift-up as $k_{x} / k_{z} \rightarrow 0$. In terms of the different components of vorticity, it is important to keep in mind that the Orr mechanism acts on the spanwise vorticity $\omega_{z}$, and that the lift-up mechanism corresponds to large production of cross-stream (or vertical) vorticity $\omega_{y}$.

Furthermore, the early-time OL-type perturbation is inherently transient, unlike the perturbations which are identified over longer time intervals. This aspect of the perturbations' character is shown in figure 2 where we plot the instantaneous growth rate

$$
\sigma(t)=\frac{1}{2 E} \frac{\mathrm{d} E}{\mathrm{~d} t}
$$

of optimal perturbations for $T=7$ (grey-dashed line, the most amplified OL-type mode marked with a black dot in figure $1 a), T=20$ for $\left(k_{x}, k_{z}\right)=\left(k_{k h}, 0\right)$ (blackdashed line, corresponds to the most unstable $k_{x}$ ), which since it corresponds to a $\mathrm{KH}$ instability we refer to as a K-type perturbation, and $T=20$ for $\left(k_{x}, k_{z}\right)=\left(k_{k h}, 0.698\right)$ and $\left(k_{x}, k_{z}\right)=\left(k_{k h}, \pi\right)$ (marked respectively by $\times$ and + in figure $1 b$, discussed in more detail in $\S 4.2$ ), which are OL-type perturbations labelled as OLE and OLH respectively, in anticipation of the elliptical and hyperbolic instabilities to which these perturbations will be related. The growth rate $\sigma$ of the $T=7$ optimal perturbation is very large at the beginning and then decays rapidly, becoming negative at about $t \sim 12$, as is typical for such transient perturbations. Conversely, the growth rate for the K-type perturbation eventually asymptotes to a finite value (the growth rate of 


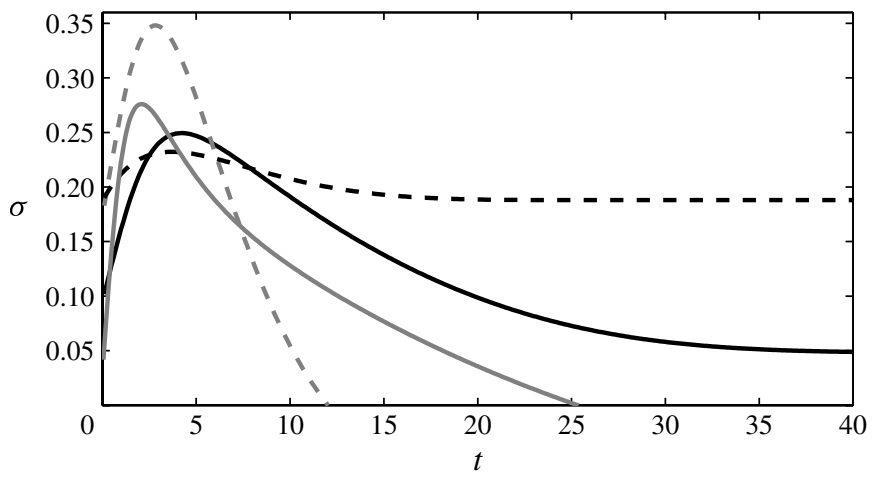

FIGURE 2. The variation of the growth rate $\sigma$ (as defined in (3.7)) with time of some representative optimal perturbations. The grey-dashed line corresponds to the optimal perturbation for $T=7$ and $\left(k_{x}, k_{z}\right)=(1.142,1.396)$ (marked with a black dot on figure $\left.1 a\right)$; the black-dashed line corresponds to the optimal perturbation for $T=20$ and $\left(k_{x}, k_{z}\right)=$ $\left(k_{k h}, 0\right)$ (marked with a black dot on figure $\left.1 b\right)$; the black line corresponds to the OLE perturbation for $T=20$ and $\left(k_{x}, k_{z}\right)=\left(k_{k h}, 0.698\right)$ (marked with $\times$ on figure $\left.1 b\right)$, and the grey line corresponds to the OLH perturbation for $T=20$ and $\left(k_{x}, k_{z}\right)=\left(k_{k h}, \pi\right)$ (marked with + on figure $1 b$ ).
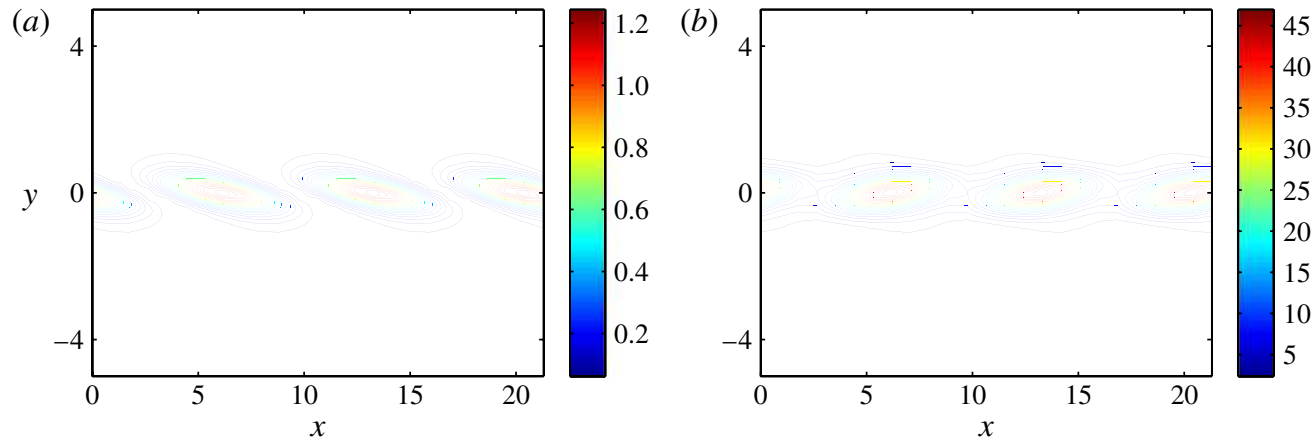

FIGURE 3. Energy density in the $(x, y)$-plane of the OLE optimal perturbation for $T=20$ and $\left(k_{x}, k_{z}\right)=\left(k_{k h}, 0.698\right)$ at $(a) t=5$ and $(b) t=15$.

the modal $\mathrm{KH}$ instability). Initially however, this perturbation also shows a slight non-modal enhancement of the (instantaneous) growth rate, associated with the perturbation extracting energy transiently via the Orr mechanism, demonstrating that even for normal modes, transient effects can lead to enhanced gain.

Finally, the 3D longer-time-interval OL-type perturbations labelled OLE and OLH show both early-time strong transient growth (with an earlier and stronger peak for OLH but larger growth up to $t=T=20$ for OLE), and much smaller (but for OLE still positive) growth rate at later times. The positive growth rate of the OLE perturbation at late times is consistent with the sub-dominant mode of the $\mathrm{KH}$ instability existing at its corresponding wavenumbers $\left(k_{x}, k_{z}\right)=\left(k_{k h}, 0.698\right)$. Figure 3 shows the energy density of the same perturbation labelled OLE at times $t=5$ and $t=15$. This quantity is normalized such that its integral over the $(x, y)$ domain containing one wavelength is one at $t=T_{0}=0$. At $t=5$, the optimal perturbation is 
oriented slightly against the shear, and for $t=15$ it has been tilted, as is characteristic of short-time optimal perturbations, at least partially subject to the Orr mechanism. The smaller longer-time growth rate for this perturbation is also consistent with the classic Squire transformation (Squire 1933) which demonstrates that oblique 3D disturbances are equivalent to 2D disturbances in a flow with a lower $R e$ (and hence lower growth rate). In the light of all these characteristics, we call this perturbation OL-type because most of the growth up to $t=20$ corresponds to non-modal growth.

These observations are entirely consistent with previous work, and in particular do not show evidence of either elliptical instability or hyperbolic instability (henceforth referred to as E-type and H-type perturbations for consistency with the OL-type and K-type nomenclature introduced above) which numerical simulation and laboratory experiment suggest are essential characteristics of transition in inflectional shear layers. Of course, this is largely unsurprising as a parallel base state has no elliptical or hyperbolic points. Indeed, it seems entirely reasonable that the primary billow, or a developing nonlinear K-type perturbation, will act in some sense as a catalyst for transition by encouraging the development of E-type or H-type perturbations. Klaassen \& Peltier (1991) and subsequently Smyth \& Peltier (1994) investigated the stability properties of an essentially fully developed primary billow. Here, we aim to extend their results to investigate what effect the developing primary billows have on the growth of secondary optimal perturbations, and indeed whether E-type and/or H-type perturbations are optimal in maximizing the perturbation energy gain over finite time horizons, which are still sufficiently long to allow the saturation of the base flow into an array of primary billows. The OL-type perturbations, being optimal for short times on the parallel flow, are strong candidates to play a leading role in the early development (when the billow has not yet developed to large amplitude) of the longer-time-interval optimal perturbations. To investigate whether the OL-type perturbations can be related to the appearance of E-type or H-type perturbations, one should consider the natural, nonlinear evolution of the K-type perturbation giving rise to the primary billow. Therefore, in the next section, we describe the properties of just such a non-parallel, time-dependent yet inherently 2D 'base' flow, whose non-modal stability properties we can then analyse.

\section{Transient response of K-type time-dependent flow}

\subsection{Base flow}

To generate a non-parallel, time-dependent 2D K-type base flow, we perform a sequence of fully nonlinear DNS of 2D perturbations of the hyperbolic-tangent parallel base flow discussed in the previous section. The size of the computational domain in the streamwise direction is set to $L_{x}=14.2$, (i.e. $k_{x}=0.4425=k_{k h}$ ) which corresponds to one wavelength of the most unstable eigenmode of K-type. In particular, this size of computational domain, along with periodic boundary conditions, suppresses the possibility of subharmonic mergings. It also restricts the possible choices of streamwise wavenumber to (integer) multiples of $k_{k h}$. From the numerical evidence this is unlikely to be too restrictive, although from figure 1, the streamwise wavenumber of the most unstable OL-type perturbation is substantially larger than $k_{k h}$. The number of gridpoints in the $x$-direction is $N_{x}=256$. The number of points in the vertical cross-stream $y$-direction is $N_{y}=512$ and the corresponding box size is $L_{y}=25$, which we believe (see for example Hazel 1972) is sufficiently large for the evolution of the primary KH billow not to be affected significantly by the vertical boundaries. 


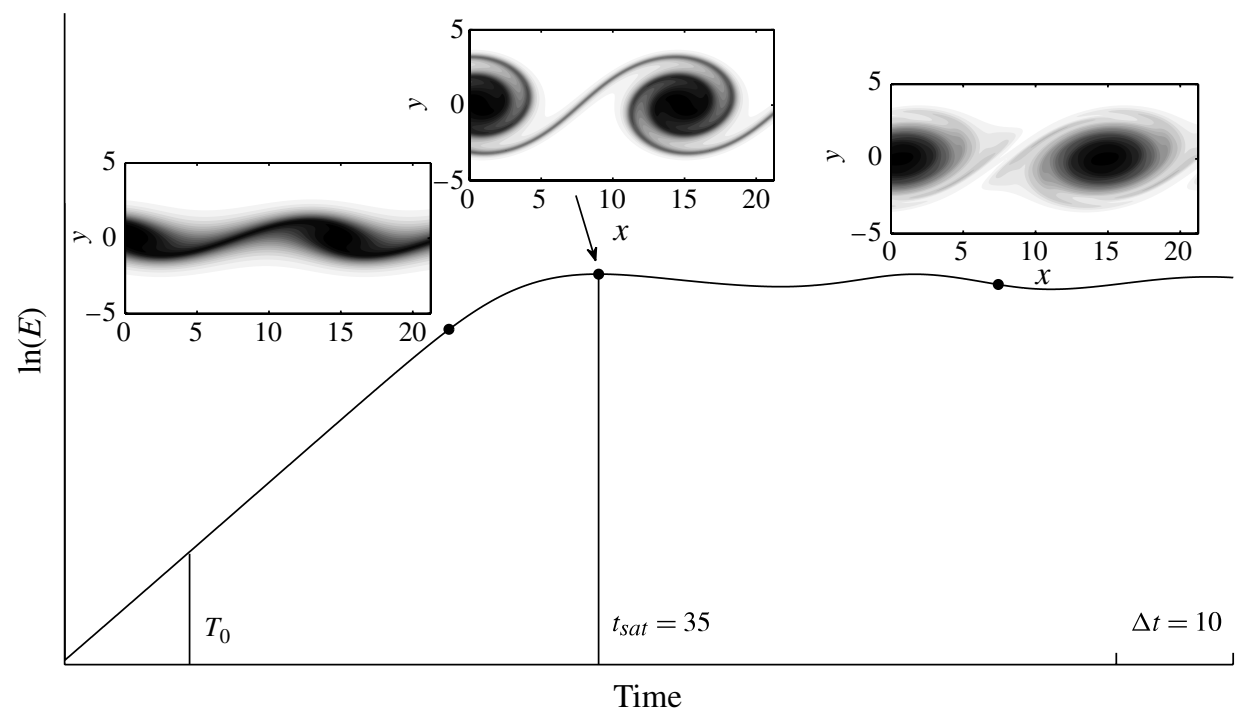

FIGURE 4. The evolution of the base flow state with time. Vertical units are arbitrary. Base flow vorticity is shown from left to right at times $t=22.2, t=t_{\text {sat }}=35$ and $t=69.2$, corresponding to the signs on the curve.

The DNS is initialized with random perturbations with zero mean and small amplitude. The initial amplitude is chosen small enough so that, after the initial transients, the most unstable mode appears long before nonlinear effects are noticeable. However, the procedure we choose to construct the base flow subsequently used in our stability analysis is a little involved, owing to the complicating effect of the diffusion of the inflectional shear layer due to the finite value of the flow Reynolds number. As a first step in this procedure, we simulate a flow with the body force (3.2) which 'freezes' the inflectional shear layer included in the governing (nonlinear) Navier-Stokes equations. The curve in figure 4 shows part of the evolution in time of the perturbation energy $E$, in this first reference simulation with the body force. In figure $4, \ln (E)$ grows linearly in time at first (at a rate entirely consistent with (twice) the growth rate of a K-type modal instability), then begins to saturate, reaches a maximum and finally oscillates slightly. Those stages correspond to the exponential growth of energy due to the $\mathrm{KH}$ instability and the subsequent nonlinear saturation leading to the roll-up of a non-stationary $\mathrm{KH}$ billow (see for example Corcos \& Sherman 1984). The saturation time $t_{\text {sat }}$ of the primary billow naturally depends on the amplitude of the initial condition and the Reynolds number of the flow, and to have a fixed time reference for all our optimization calculations, we arbitrarily set $t_{\text {sat }}=35=t_{\text {sat-bf }}$ (for base flow). We refer to the entire time-dependent 2D flow obtained from this reference simulation with the body force as $\boldsymbol{U}_{b f}(t)$. Because of the body force present in its computation, the relevant $\boldsymbol{U}_{b f}$ can be easily reproduced with any initial condition with sufficiently small amplitude and a subsequent re-definition of the time origin such that $t_{\text {sat }}=35$.

We then conduct other simulations without the body force (and thus solutions to the actual full nonlinear Navier-Stokes equations). Each simulation is constructed for a specific start time $T_{0}$ of the optimization time interval, and is also specifically tailored so that the primary billow still saturates at a time $t_{s a t} \simeq t_{\text {sat-bf }}=35$. For an optimization 


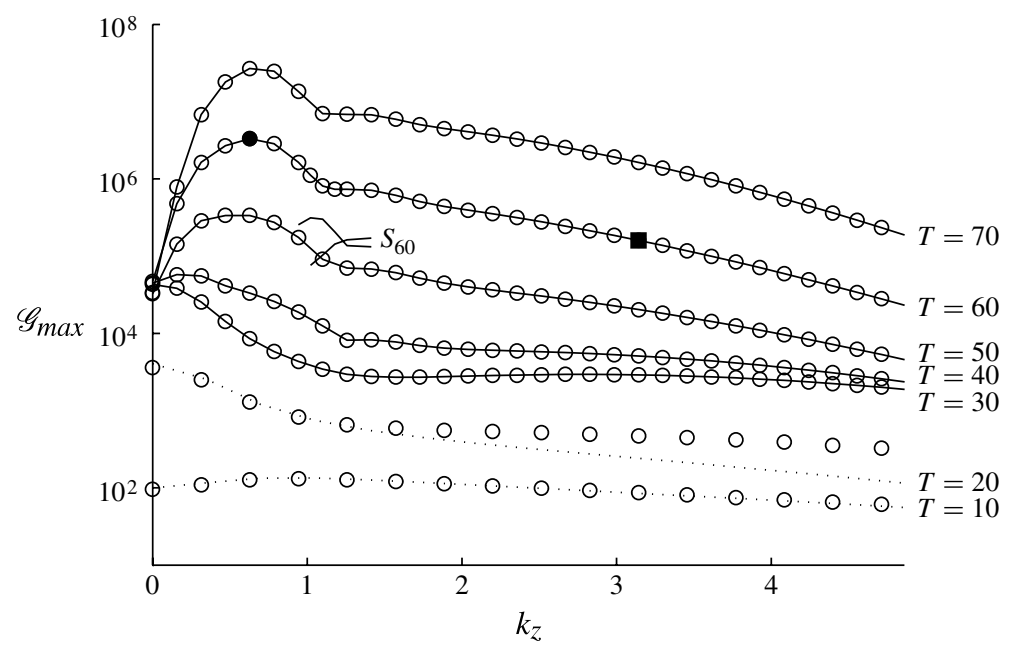

FIGURE 5. Optimal gain versus $k_{z}$ for different optimization times as indicated on the figure with an optimization interval starting at $T_{0}=0$. For comparison the dotted lines show the results for $T=10,20$ of the primary instability presented in $\S 3$. The $\boldsymbol{0}$ and $\mathbf{\square}$ symbols on the $T=60$ curve correspond respectively to the E-type and H-type perturbations subsequently described in more detail. For all curves, the $k_{x}=k_{k h}=0.4425$, except for the dotted line for $T=10$, where $k_{x}=2 k_{k h}$. The lines marked $S_{60}$ are segments of two subdominant branches for $T=60$.

interval starting at $T_{0}$, a DNS without the body force is initialized with $\boldsymbol{U}_{b f}\left(T_{0}-\delta t\right)$ at time $t=T_{0}-\delta t$. In this new DNS, the saturation time $t_{\text {sat }}$ would in general occur at a time slightly different from the reference time $t_{\text {sat-bf }}=35$, say $35+t_{-v}$. We tune the offset time $\delta t$ used for initializing the DNS until $0<t_{-v}<1$. We then reset the zero of time so that we obtain a $2 \mathrm{D}$ flow $\boldsymbol{U}(t)$ with no body force from $T_{0}-t_{-v}$ which thus saturates, similarly to the reference flow (with a body force) at $t_{\text {sat }}=t_{\text {sat-bf }}=35$. As a specific example, the insets of figure 4 show vorticity snapshots of $\boldsymbol{U}(t)$ for $T_{0}=0$ and $t_{-v}=0.8$. Therefore, the evolving base flow always reaches its maximum amplitude at $t=t_{\text {sat }}$. Although formally the algorithm requires $\boldsymbol{U}(t)$ at every time step, for computational efficiency, we save the full flow field $\boldsymbol{U}(t)$ and then linearly interpolate it every 2 time units. We then use this linearly interpolated base flow for our optimization calculations. Interpolating the base flow every time unit required shorter time stepping in the optimization calculations but provided similar results.

\subsection{Optimal perturbations from $T_{0}=0$ : E-type and H-type}

A natural place to start in our consideration of linear perturbation growth on a timeevolving base flow susceptible to a primary $\mathrm{KH}$ instability is to consider optimization time intervals starting very early in the flow evolution, and thus potentially including (for sufficiently long intervals) the roll-up before saturation of the primary K-type instability. Figure 5 shows $\mathscr{G}_{\max }$ for $T_{0}=0$ and different $T$. For all these curves the minimal streamwise wavenumber is constrained to be $k_{x}=k_{k h}$, but all integer multiples are allowed. Also indicated is the gain of the optimal perturbations for the frozen (by a body force) tanh profile (as discussed in $\S 3$ ) at $k_{x}=k_{k h}$ for $T=20$ and at $k_{x}=2 k_{k h}$ for $T=10$. (Choosing this increased streamwise wavenumber is consistent with figure 1 for the early-time-interval OL-type perturbations.) For these short optimization times $(T \lesssim 20)$, optimal energy growth resembles that of the parallel, frozen hyperbolic- 
tangent profile considered in $\S 3$ (except for large $k_{z}$ in which case the optimal perturbations concentrate more around the incipient braid). In particular, the mostamplified optimal perturbation is clearly 3D for $T=10$ and $2 \mathrm{D}$ for $T=20$, entirely consistent with figure 1 . For $T=30$, the most amplified optimal perturbation is still $2 \mathrm{D}$, yet for $T \gtrsim 50$, the most amplified optimal perturbation is definitely inherently 3D, and occurs for a particular spanwise wavenumber $k_{z} \approx 0.6$.

It is apparent that for sufficiently long optimization times, $T \gtrsim 50$, the structure of the gain's dependence on $k_{z}$ changes qualitatively, and there appears to be a new perturbation type appearing centred around $k_{z} \simeq 0.6$. It could seem that the abrupt change of slope of $\mathscr{G}_{\max }$ at the end of the peaked area around $k_{z} \simeq 1$ is due to the cross-over of branches corresponding to different modes of the composed directadjoint propagator $\left(\Phi_{\lambda}^{\dagger} \Phi_{\lambda}\right.$ in $\left.\S 2\right)$. A Krylov method similar to that described in Donnadieu et al. (2009) was used to approximate the leading subdominant modes of the direct-adjoint propagator for $T=60$. (The convergence of the results was checked by using different Krylov subspaces typically of dimension five or six.) The two leading subdominant branches were followed around $k_{z} \simeq 1$ (where the slope of $\mathscr{G}_{\max }$ changes abruptly) and are indicated as $S_{60}$ in figure 5, showing that there is actually no cross-over involving the branch of the optimal perturbations. It turns out that for $T=60$ (and plausibly for $T \gtrsim 50$ ) the leading branch and the corresponding optimal perturbations are strongly dependent on $k_{z}$ in the region $1 \lesssim k_{z} \lesssim 1.8$, just after the peak. Since this time interval corresponds to times definitely longer than the saturation time, and so optimization intervals when the primary billow has definitely reached finite amplitude, analysis of perturbation structure both in this peaked area and in the qualitatively different higher wavenumber tail is clearly of interest.

As already noted, the particular different types of perturbation (elliptical E-type or hyperbolic H-type) can be characterized by their spatial location and the relative vorticity distribution. Although the definition of being core-centred or braid-centred is naturally somewhat arbitrary, we have found it useful to consider how the fraction of spanwise vorticity with respect to total enstrophy, i.e. $\int \omega_{z}^{2} \mathrm{~d} V / \int|\omega(t)|^{2} \mathrm{~d} V$, varies for perturbations with different spanwise wavenumbers $k_{z}$, and different optimization intervals starting at $T_{0}=0$. This quantity is shown in figure 6 for the different optimization intervals starting at $T_{0}=0$. We expect E-type responses to be associated with relatively large spanwise $\omega_{z}$ components, while $\mathrm{H}$-type perturbations should be associated with relatively large vertical $\omega_{y}$ and/or streamwise $\omega_{x}$ components, which reflects directly in a lower $\omega_{z}$ fraction. Interpreted in this way, the data shown in the figure suggest that smaller- $k_{z}$-wavenumber perturbations may be E-type, while larger- $k_{z}$-wavenumber perturbations may well be H-type.

This suggestion is reinforced by consideration of the spatial compartmentalization of the perturbation kinetic energy. Once again, this is somewhat arbitrary, but we define the 'core' of the primary K-type billow to be the fluid included within an ellipse which best fits the contour containing the spanwise vorticity equal to or greater than $70 \%$ of the instantaneous maximum of the spanwise vorticity in the primary K-type billow. We then identify a particular optimal perturbation as being of core-centred E-type if $25 \%$ or more of the perturbation kinetic energy is contained within this 'core contour ellipse'. On the same figure 6, we plot with grey circles the fraction of the perturbation kinetic energy contained within this core contour ellipse, and mark with shading those perturbations which we identify as being of E-type (i.e. where this fraction is larger than 0.25). Over all the shown optimizing intervals (all starting at $\left.T_{0}=0\right)$ smaller spanwise wavenumbers $k_{z}$ are then classified as being of E-type, while higher wavenumbers appear to be more plausibly described as being of H-type 


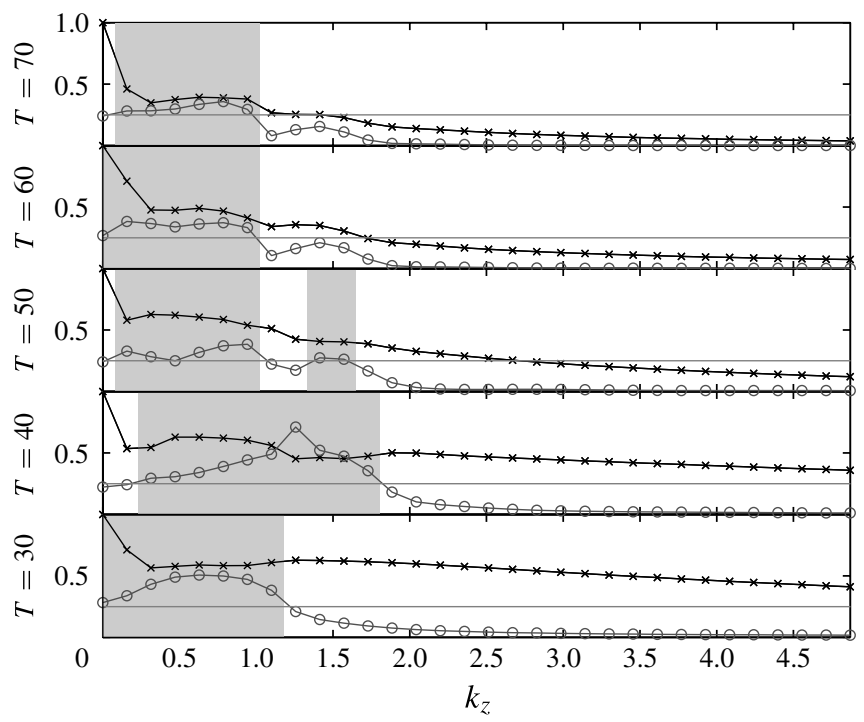

FIGURE 6. The black line with $\times$ symbols shows the $k_{z}$ dependence of the fraction of spanwise vorticity $\omega_{z}$ with respect to total enstrophy $\int \omega_{z}^{2} \mathrm{~d} V / \int|\omega(t)|^{2} \mathrm{~d} V$ for the optimal responses from $T_{0}=0$. The grey line with $\bigcirc$ symbols shows the energy fraction inside the ellipse that best fits the contour corresponding to 0.7 of the instantaneous maximum base flow vorticity. When this fraction is larger than 0.25 (shown with the grey horizontal line) we identify the response as being of E-type, which is shaded on the figure. Indicated beside each plot is the final time $T$ of the optimization interval.

(particularly noticeably the higher $k_{z}$ perturbations have virtually no kinetic energy in the primary K-type billow core).

To investigate in more detail whether these classifications are appropriate, we consider the development of four specific perturbations in detail. We describe the development of the optimal perturbation for $T=60$ and $k_{z}=\pi / 5$ (wavenumber of the most amplified perturbation at $T=60$ ), marked with a black dot on figure 5, which within our classification we expect to be of elliptical E-type. This perturbation has very similar values of streamwise and spanwise wavenumbers $\left(\left(k_{x}, k_{z}\right)=\left(k_{k h}, \pi / 5\right)\right)$ to the OL-type perturbation optimized over a short interval with $T=20$ on the frozen parallel hyperbolic-tangent flow $\left(\left(k_{x}, k_{z}\right)=\left(k_{k h}, 0.698\right)\right)$ labelled as OLE and shown in figure 1. As we shall see, the early-time behaviour of the most amplified perturbation for the longer time interval with $T=60$ actually has many points of similarity with the OLE perturbation. Since the higher wavenumber 'tail' is also of interest, and appears to be (at least potentially) of H-type, we also then consider a perturbation with $k_{z}=\pi$ for the same optimizing interval, i.e. with $T_{0}=0$ and $T=60$, and compare it with the OLH perturbation that has the same wavenumbers.

\subsection{E-type response with maximum growth rate for $T_{0}=0, T=60$}

The optimal perturbation for $T=60$ with $k_{z}=\pi / 5$ has the maximum gain over this time interval of all possible perturbations. However, since we have a complete numerical simulation of its evolution during this optimization interval, we can also identify the instantaneous growth rate of the perturbation at all times. Indeed, by consideration of the perturbation kinetic energy, it is straightforward to derive an evolution equation for this growth rate. Taking the scalar product of $\boldsymbol{u}$ with the 
momentum equation (2.1a), integrating on the $(x, y)$-plane and arranging terms we have

$$
\begin{aligned}
\frac{\mathrm{d} E}{\mathrm{~d} t}=- & \overbrace{\int u v \frac{\partial \bar{U}}{\partial y} \mathrm{~d} x \mathrm{~d} y}^{\text {Mean shear }}-\overbrace{\int u v\left(\frac{\partial U_{2 D}}{\partial y}+\frac{\partial V_{2 D}}{\partial x}\right) \mathrm{d} x \mathrm{~d} y}^{\text {Shear } 2 \mathrm{D}} \\
& -\underbrace{\int\left(u^{2}-v^{2}\right) \frac{\partial U_{2 D}}{\partial x} \mathrm{~d} x \mathrm{~d} y}_{\text {Strain } 2 \mathrm{D}}-\underbrace{R e^{-1} \int \boldsymbol{u} \cdot \nabla^{2} \boldsymbol{u} \mathrm{d} x \mathrm{~d} y}_{\text {Dissipation }},
\end{aligned}
$$

where we have introduced

$$
\bar{U}(t)=\frac{1}{L_{x}} \int_{0}^{L_{x}} U(t) \mathrm{d} x,
$$

the streamwise-averaged (parallel but time varying) base flow and

$$
\left(U_{2 D}, V_{2 D}, 0\right)^{\mathrm{T}}=\boldsymbol{U}_{2 D}(t)=\boldsymbol{U}(t)-\bar{U} \cdot \boldsymbol{e}_{x}
$$

is the inherently non-parallel 2D flow associated with the K-type billow. This decomposition shows that there are four contributions to the growth in the energy of the secondary 3D perturbation: energy extraction from the streamwise-averaged mean (parallel) shear via the Reynolds stress; energy extraction from the non-parallel shear associated with the primary K-type billow; energy extraction from the strain field associated with the primary K-type billow; and dissipation, which of course always acts as a sign-definite sink of perturbation kinetic energy. (See for example Caulfield \& Peltier 2000 for a more detailed discussion of this decomposition.)

In figure $7(a)$ we plot the instantaneous growth rate $\sigma(t)$ and these various components for the (presumed E-type) optimal perturbation for $T=60$ with $k_{z}=\pi / 5$. Although this perturbation grows across the entire optimization interval, it is clear that the largest growth rate occurs at the beginning of the flow evolution. We also plot (with a dashed line) the instantaneous growth rate for the OLE perturbation until $t=20$, essentially a replotting of the data shown in figure 2. Clearly the optimal perturbation behaves initially very similarly to the OLE perturbation (the corresponding dashed line being barely distinguishable from the thick line), and this strong initial growth is thus associated with a (transient) exploitation of the Orr and lift-up mechanisms before the primary K-type billow has rolled up. Unsurprisingly, in terms of energetics, the dominant driving mechanism is extraction from the streamwise-averaged shear, which though initially strongest, continues to occur throughout the subsequent (base flow) evolution when the primary billow has rolled up.

Furthermore, the spatial distribution of the perturbation confirms that this perturbation is appropriately described as being of elliptical E-type. In figure 8, we plot contours of the energy density for this optimal perturbation at different times. The flow evolution is now clear. At early times, the perturbation is tilted into the shear (as well as being periodically varying in the streamwise direction). Therefore, it is perfectly arranged to exploit the Orr and lift-up mechanisms to extract transient growth at early times from the quasi-parallel shear flow, almost exactly like the OLE perturbation discussed above and shown in figure 3, the main difference being that the early E-type perturbation has more energy in the braid. Since the base flow is now no longer streamwise-independent, there is now a preference for such a 

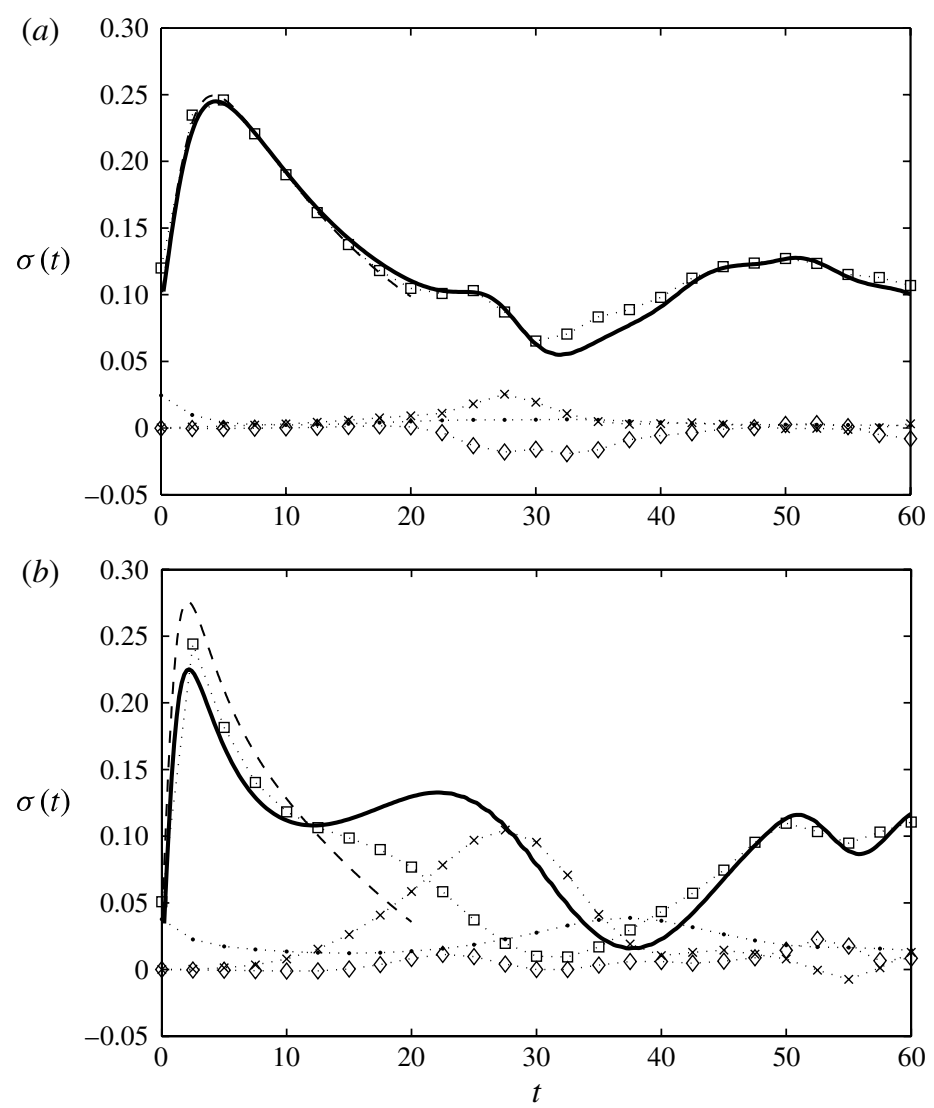

FIGURE 7. Instantaneous growth rate $\sigma(t)$ (thick continuous line, as defined in (3.7)), for the optimal perturbations from $t=0$ to $t=60, k_{z}=\pi / 5$ of the E-type mode in $(a)$, and $k_{z}=\pi$ of the H-type mode in $(b)$. Dashed lines in $(a, b)$ show respectively the growth rate of the OLE and OLH optimal perturbations of the frozen tanh profile for $T=20$. The dotted line with $\bullet$ symbols shows the dissipation and also shown are the growth rate contributions from: $\square$, the mean shear; $\diamond$, the shear $2 \mathrm{D}$ and $\times$, the strain $2 \mathrm{D}$.

perturbation to grow within the strong strain field in the vicinity of the braid. As the billow rolls up further, this essentially braid-centred perturbation is then wellplaced to perturb the billow core strongly, and trigger behaviour that is closely related to the idealized elliptical instability, and thus appropriately described as an E-type perturbation. The spanwise wavenumber of these perturbations is particularly well-suited to trigger an elliptical instability, consistently with the frozen-in-time calculations of Klaassen \& Peltier (1991) and Caulfield \& Peltier (2000). Loosely, the braid-centred perturbations have just the right wavenumber to make the elliptical core 'ring' with a translative/elliptical instability (as is apparent in the $t=25$ and $t=30$ panels) which then in turn grows strongly. Interestingly, as is apparent from figure $7(a)$, the time $(t \sim 30)$ when the braid-centred perturbation is interacting with the core to trigger the more overtly E-type behaviour is actually associated with a relatively small value of the instantaneous growth rate $\sigma$ (as defined in (3.7)), presumably due to the flow reorganizing itself as it passes from one stage of perturbation growth to another. 


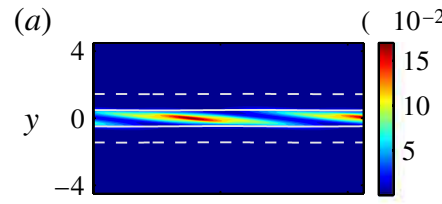

(d)

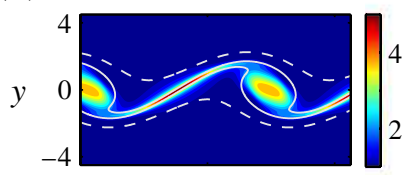

(g)

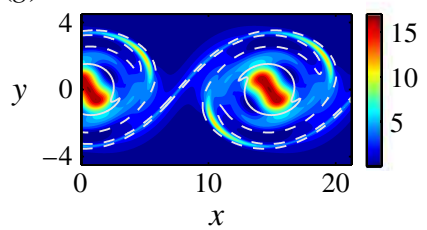

(b)

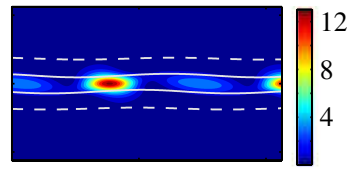

(e)

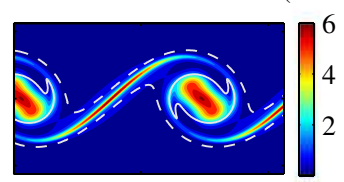

(h)

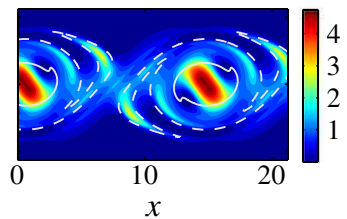

(c)

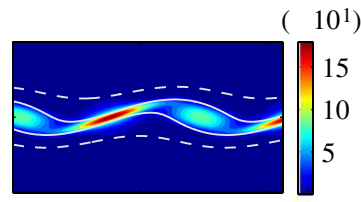

(f)

$\left(\times 10^{2}\right)$

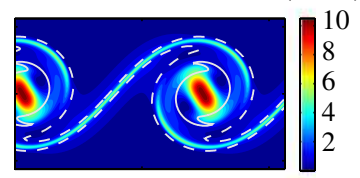

(i)

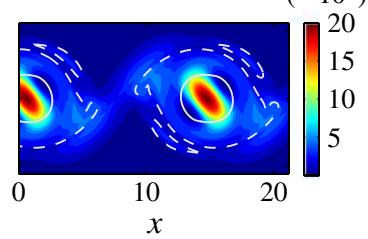

FIGURE 8. Scaled energy density of the optimal perturbation from $t=0$ to $t=60$ with $k_{x}=k_{k h}$ and $k_{z}=\pi / 5$ (E-type) at: (a) $t=0 ;(b) t=10 ;(c) t=20 ;(d) t=25 ;(e) t=30 ;(f)$ $t=35$; $(g) t=40$; (h) $t=45$; ( $i) t=60$. At $t=T_{0}=0$, the integral over the domain of the energy density is set to one. Level sets of the instantaneous base flow vorticity at $\omega_{z}=-0.8$ and $\omega_{z}=-0.2$ are plotted with a white solid line and a white dashed line respectively.

This two-stage growth process is also confirmed by considering the time-evolution of the different components of vorticity for both this E-type perturbation, and the earlytime OLE perturbation, as shown in figure $9(a)$. The evolution of the perturbation is always strongly dominated by spanwise vorticity, $\omega_{z}$ which is seeded by the early-time transient growth spurt associated with the mixture of the Orr and lift-up mechanisms experienced by the OLE perturbation on the parallel hyperbolic-tangent shear flow, and is characteristic of elliptical or translative instabilities. Finally, this concept of an OL-type Orr/lift-up mixed perturbation developing in the braid that then perturbs the billow core is reinforced by considering the $3 \mathrm{D}$ rendering of the perturbation at $t=45$, as shown in figure 10. This clearly shows that the perturbation has a strong signal in the braid, which is wrapping around the core, leading to a ringing of the primary billow core. This coupled structure is consistent with the quote from Rogers \& Moser (1992) presented in the introduction.

However, it is important to appreciate that the spanwise wavenumber of this perturbation is too small to be consistent with the rib vortices numerically observed by Caulfield \& Peltier (2000) for example, and, of course, the perturbation shown in figure 10 has been calculated in a strictly linear regime. It is also important to observe that, at least qualitatively, the spatial structure within the core of this perturbation is similar to the core-centred instability identified by Potylitsin \& Peltier (1998), as shown in their figure $9(a)$, although they assumed a strong separation in time scales between the temporal evolution of the base flow and the developing secondary perturbations. Here, the time evolution of the base flow appears to play a crucial role. The calculated gain of the optimal (in the sense of maximizing the gain) perturbation 

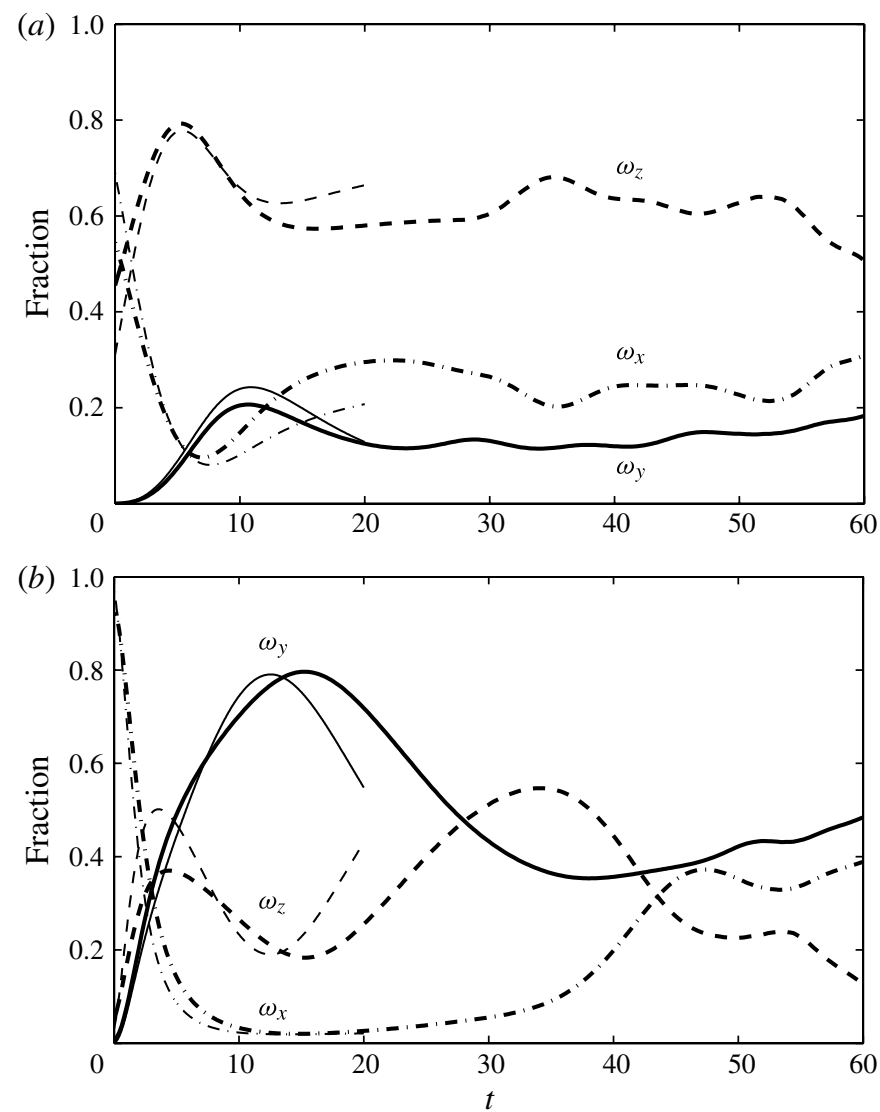

FIGURE 9. Evolution of the fraction of each vorticity component with respect to total enstrophy $\int \omega_{i}(t)^{2} \mathrm{~d} V / \int|\omega(t)|^{2} \mathrm{~d} V$ for the optimal perturbation from $t=0$ to $t=60$ with $k_{x}=k_{k h}$, and $k_{z}=\pi / 5$ (E-type) in $(a)$ and $k_{z}=\pi$ (H-type) in $(b)$. The continuous lines correspond to $\omega_{y}$, the dashed lines correspond to $\omega_{z}$ and the dash-dotted lines correspond to $\omega_{x}$. Also shown with thin lines extending up to $t=20$ are the same quantities for the OLE $(a)$ and OLH $(b)$ optimal perturbations of the frozen tanh profile.

is substantially larger than that of perturbations with larger $k_{z}$, and the spatial structure of this optimal perturbation also has substantial streamwise vorticity $\omega_{x}$ in the braid.

Nevertheless, it appears that using the tools of non-modal stability theory leads to the natural identification of 'optimal' perturbations which have many of the characteristics of core-centred elliptical instabilities, with the added insight that such E-type perturbations are actually very strongly excited by OL-type behaviour at early times. Fundamentally, the temporal sequence of distinct stages of growth of this infinitesimal perturbation relies on and exploits the strongly time-dependent nature of the evolving base flow, and so the assumption that either a frozen-in-time approximation, or indeed even some time-averaging of the base flow can capture the central dynamics and growth mechanisms for this perturbation does not appear to be supported by our calculations. Furthermore, by comparison of figures 1 and $7(a)$, there is no real scale separation between the growth rates of the E-type perturbations (typically greater than 0.1 until the primary billow saturates) and the 


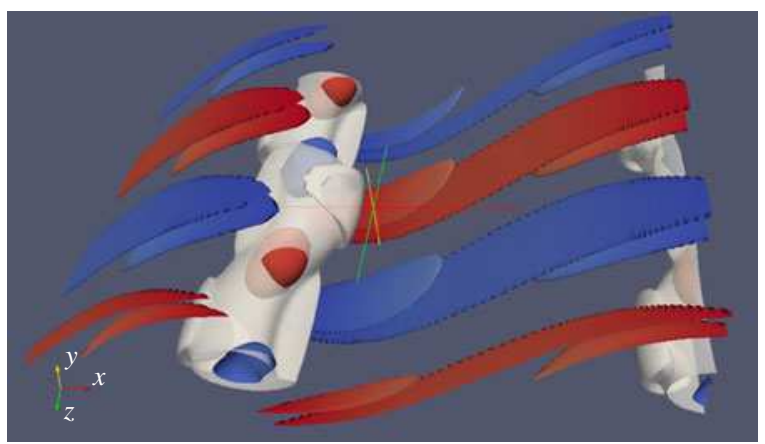

FIGURE 10. E-type optimal perturbation at time $t=45$ superposed on the base flow. The perturbation amplitude is such that the maximum value of the $\omega_{z}$ vorticity (spanwise) is 0.4 . The white semi-transparent contour corresponds to the isocontour $\omega_{z}=-0.9$. The red/blue contours correspond to $\left(\omega_{x}^{2}+\omega_{y}^{2}\right)^{1 / 2}=0.2$, the colouring being according to the magnitude of $\omega_{x}$, red (respectively blue) representing positive (negative) values of $\omega_{x}$.

growth rate of the primary K-type perturbation (somewhat less than 0.2 ). Therefore, despite the useful insights that the frozen-in-time analysis provides for characterizing the secondary instability mechanisms that could prevail at late times, it does seem necessary to consider the time-dependence of the base flow explicitly in determining the properties of the optimal secondary perturbations. However, the strong excitation of E-type behaviour by OL-type behaviour at early times naturally raises the question as to how sensitive the development of such E-type perturbations is to the chosen start time $T_{0}$ for the optimization interval. We return to this question after considering the properties of the higher wavenumber H-type optimal perturbations.

\subsection{H-type response for large $k_{z}$}

The behaviour is, in many important aspects, qualitatively different for the perturbation at higher wavenumber, as shown in figures $7(b), 11$ and $9(b)$, equivalent to their E-type counterparts. Considering the instantaneous growth rate first, this perturbation also experiences an early-time enhanced growth from the Orr and lift-up mechanisms, extracting energy from the mean shear. The characteristic tilting over of the perturbation by the mean shear is clearly apparent in the early-time panels of figure 11 showing the energy density distribution of the perturbations. Initially, this growth is naturally dominated by energy extraction from the mean shear. However, the physical character of this perturbation is very different, as this early-time evolution is dominated by the expected vertical vorticity $\omega_{y}$ as shown in figure $9(b)$. This is entirely consistent with a larger contribution from the lift-up mechanism that can be expected for this larger $k_{z}$, as described in $\S 3$.

This structural difference ensures that the subsequent evolution (once the primary K-type billow is saturated) is also qualitatively different. There is a substantial and essential contribution to growth at times around $t \sim 30$ from the strain field, as these vertical vortices are stretched and tilted by the strain field at the hyperbolic points in the braid and wrapped around the primary billow cores. As is apparent in the later-time energy panels (and through comparison with for example figures 7, 13 and 14 of Caulfield \& Peltier 2000) this perturbation is strongly localized in the braid region, has much of the character of both the hyperbolic instability and also the finite-amplitude development of rib vortices. Indeed, the spanwise wavenumber for this 
(a)

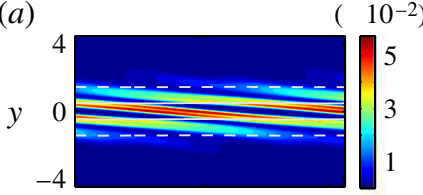

(d)

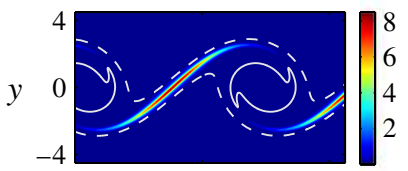

(g)

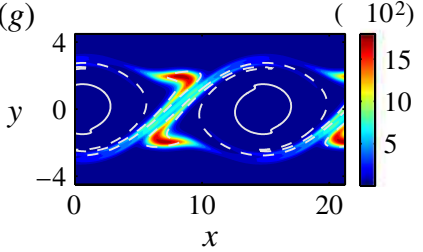

(b)

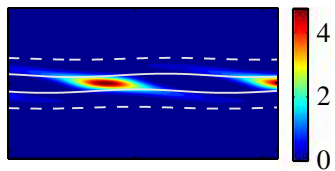

(e)

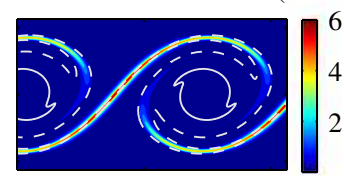

(h)

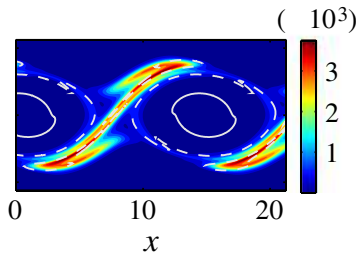

(c)

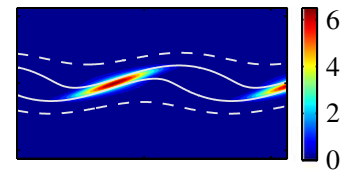

(f)

$\left(\times 10^{2}\right)$

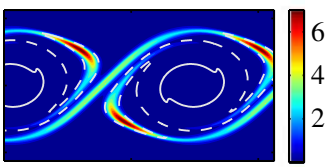

(i)

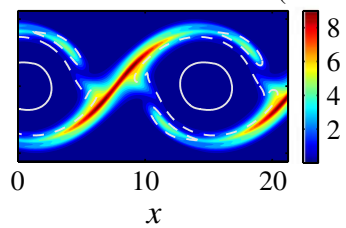

FIGURE 11. Scaled energy density of the optimal perturbation from $t=0$ to $t=60$ with $k_{x}=k_{k h}$ and $k_{z}=\pi$ (H-type) at: (a) $t=0 ;(b) t=10 ;(c) t=20 ;(d) t=30 ;(e) t=40 ;(f)$ $t=45$; (g) $t=50$; (h) $t=55$; (i) $t=60$. At $t=T_{0}=0$, the integral over the domain of the energy density is set to one. Level sets of the instantaneous base flow vorticity at $\omega_{z}=-0.8$ and $\omega_{z}=-0.2$ are plotted with a white solid line and a white dashed line respectively.

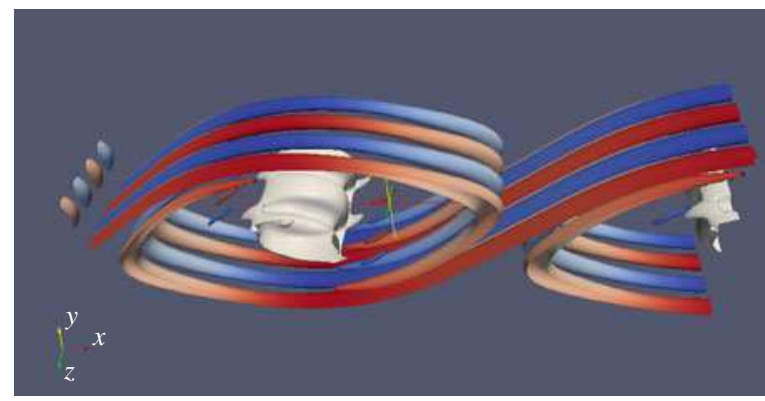

FIGURE 12. H-type optimal perturbation at time $t=45$ superposed on the base flow. The perturbation amplitude is such that the maximum value of the $\omega_{z}$ vorticity (spanwise) is 0.4. The white contour corresponds to the isocontour $\omega_{z}=-0.9$. The red/blue contours correspond to $\left(\omega_{x}^{2}+\omega_{y}^{2}\right)^{1 / 2}=0.2$, the colouring being according to the magnitude of $\omega_{x}$, red (respectively blue) representing positive (negative) values of $\omega_{x}$.

perturbation is much closer to that typically observed for rib vortices than for the optimal E-type perturbation discussed above. The wavenumber is also too large for the inevitable disruption of the core to trigger a significant E-type perturbation. The $\mathrm{H}$-type perturbation does wrap around the core, as is shown in panels for $t \simeq 50$ in figure 11 , when there is reinjection of vorticity associated with enhanced instantaneous perturbation growth rate $\sigma$ (see figure $7 b$ ), but there is still no significant energy density for the perturbation in the elliptical core. This picture is further reinforced by considering the $3 \mathrm{D}$ rendering of this $\mathrm{H}$-type perturbation at $t=45$ (figure 12), 


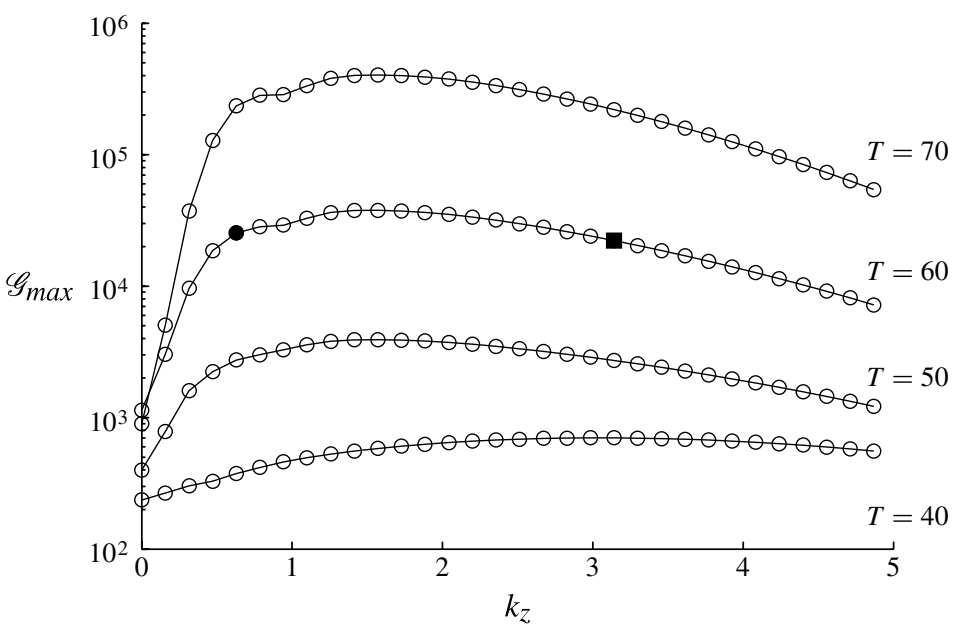

FIGURE 13. Optimal gain versus $k_{z}$ for different optimization times as indicated on the figure, with an optimization interval starting at $T_{0}=20$. The and $\mathbf{\square}$ symbols on the $T=60$ curve correspond respectively to the E-type and H-type perturbations subsequently described in more detail. For all curves, $k_{x}=k_{k h}=0.4425$.

which shows the much smaller wavelength of the streamwise vortices compared to those shown in figure 10, and the absence of any significant perturbation in the primary billow core. The spatial structure of this perturbation seems consistent with the braid-centred instability identified by Potylitsin \& Peltier (1998), as shown in their figure $9(b)$.

Therefore, we believe that this perturbation can appropriately be described as being of H-type, although it is important to appreciate that as yet no causal relationship between this (inherently linear) perturbation and the profoundly nonlinear rib vortices has been established. Nevertheless, it has been shown that E-type and H-type perturbations naturally arise from a non-modal optimal calculation, consistently (for the H-type perturbation at least) with previous simplified models (see for example Caulfield \& Kerswell 2000). Furthermore, our calculations appear to suggest that the two types of perturbations are distinct classes of perturbations, utilizing qualitatively different growth mechanisms, both in terms of spatial vorticity and energy distribution and source of energy for growth. We now consider a time interval with a later start time, (i.e. $T_{0}>0$ ) in an attempt to investigate how important the initial OL-type perturbation growth is to the ultimate character of the optimal perturbations.

\section{Variation in optimization-interval start time $T_{0} \neq 0$}

In this section we choose $T_{0}=20$, in an attempt to remove the influence of any initial period where the evolving base flow is close to parallel. This variation in start time also removes much of the initial stage of base flow evolution where the base flow itself is rapidly evolving as the primary billows roll up, thus making it more likely that an assumption that the base flow state is frozen in time is a defensible, albeit still approximate, approach for the identification of secondary growing perturbations. As shown in figure 4 , by $t \simeq 20$, the base flow is strongly non-parallel and nonlinear, though the primary K-type billow has not saturated at its maximum amplitude. Essentially repeating the analysis of $\S 4.2$, in figure 13 , we plot 


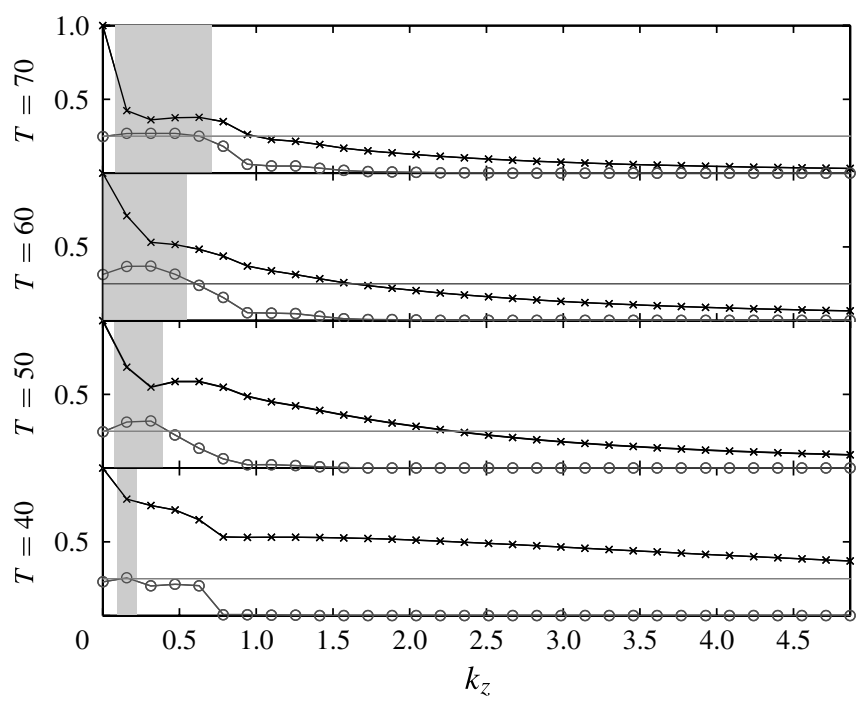

FIGURE 14. The black line with $\times$ symbols shows the $k_{z}$ dependence of the fraction of spanwise vorticity $\omega_{z}$ with respect to total enstrophy $\int \omega_{z}^{2} \mathrm{~d} V / \int|\omega(t)|^{2} \mathrm{~d} V$ for the optimal responses from $T_{0}=20$. The grey line with $\bigcirc$ symbols shows the energy fraction inside the ellipse that best fits the contour corresponding to 0.7 of the instantaneous maximum base flow vorticity. When this fraction is larger than 0.25 (shown with the grey horizontal line) we identify the response as being of E-type, which is shaded on the figure. Indicated beside each plot is the final time $T$ of the optimization interval.

optimal gain against $k_{z}$ for different final times $T$, for optimization intervals with start time $T_{0}=20$. (We note that slow convergence to the optimal perturbation is observed for a few small values of $k_{z}$ in the $T=40$ curve; we use the Krylov method mentioned in $\S 4$ to obtain the optimal gain.) By comparison with the equivalent figure 5 for optimizations with $T_{0}=0$, it is apparent that the gain is (unsurprisingly) reduced across all the wavenumbers, but most significantly for the relatively low-wavenumber peak which has now been identified as being characteristic of E-type perturbations. This is evidence that the strong growth associated with initial OL-type behaviour has been at least partially suppressed by removing the initial time period when the Orr and lift-up mechanisms are most efficient in causing energy gain.

\subsection{E-type response}

This picture of relative suppression of E-type behaviour is reinforced by consideration of the compartmentalization of kinetic energy and vorticity in figure 14. By comparison with the equivalent figure for $T_{0}=0$, (i.e. figure 6), it is clear that E-type behaviour is significantly rarer, and over much more of the wavenumber range both the vorticity and energy distributions have more of the characteristics of H-type perturbations. In particular, at the specific marked value of $k_{z}=\pi / 5$ for a perturbation optimized over the interval $20 \leqslant t \leqslant 60$, consideration of the instantaneous growth rate in figure 15, and the perturbation kinetic energy in figure 17 show that at least initially the perturbation has certain H-type aspects. After a substantially weaker (compared to the perturbation from a flow with $T_{0}=0$ ) initial Orr mechanism 'tilting', there is an early-time contribution to the growth rate from the strain field (around $t \simeq 20-30$ ). This contribution leads to much stronger energy density in the braids, which only 


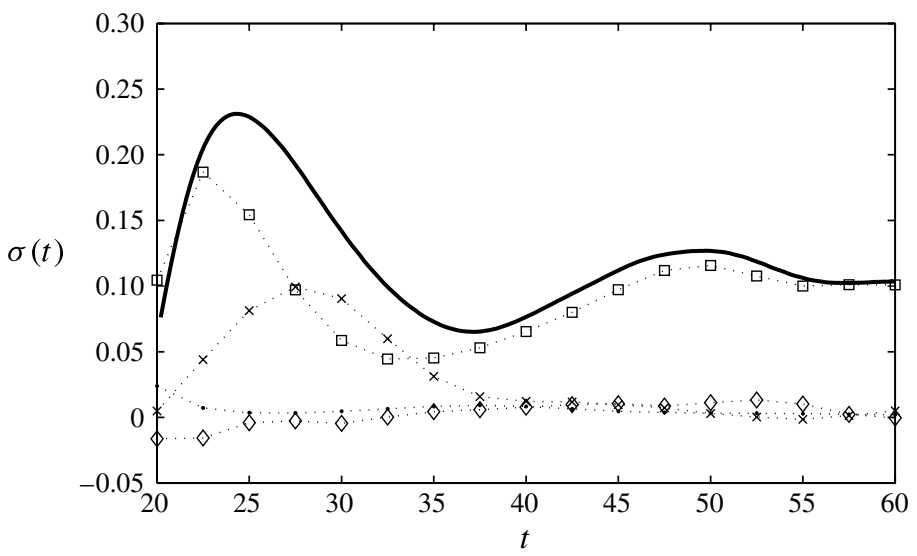

FIGURE 15. Instantaneous growth rate $\sigma(t)$ (thick continuous line, as defined in (3.7)) of the optimal perturbation from $t=20$ to $t=60$ and $k_{z}=\pi / 5$ of the E-type mode. The dotted line with $\bullet$ symbols shows the dissipation and also shown are the growth rate contributions from: $\square$, the mean shear; $\diamond$, the shear $2 \mathrm{D}$ and $\times$, the strain $2 \mathrm{D}$.

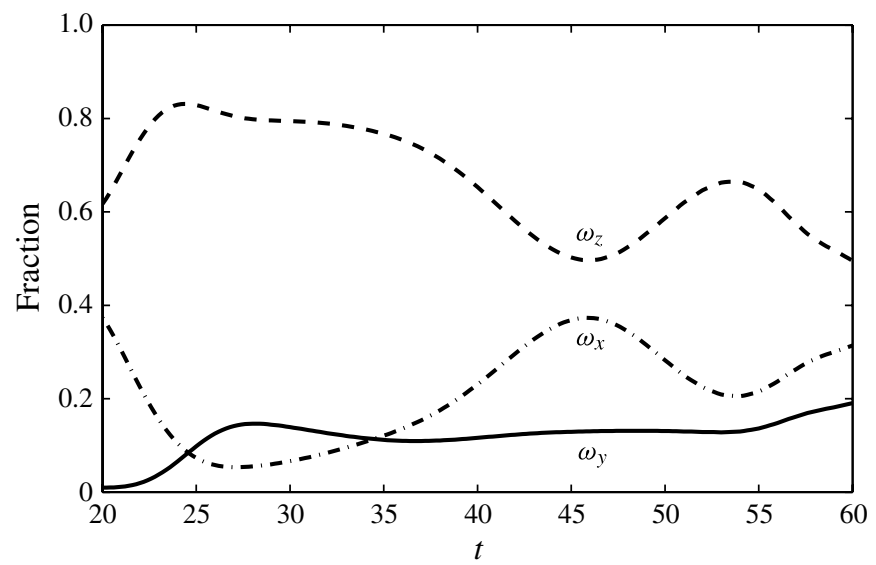

FIGURE 16. Evolution of the fraction of each vorticity component with respect to total enstrophy $\int \omega_{i}(t)^{2} \mathrm{~d} V / \int|\omega(t)|^{2} \mathrm{~d} V$ for the optimal perturbation from $t=20$ to $t=60$ with $k_{x}=k_{k h}$ and $k_{z}=\pi / 5$ (E-type). The continuous line corresponds to $\omega_{y}$, the dashed line corresponds to $\omega_{z}$ and the dash-dotted line corresponds to $\omega_{x}$.

eventually leads to strong perturbation energy in the billow core (by $t \simeq 60$ ) which is more characteristic of E-type behaviour. (There is once again a drop in instantaneous perturbation energy growth rate $\sigma$ apparent in figure 15 as the perturbation starts to switch from being in the braid to being in the core.) This is consistent with our previous description that a key aspect of the development of an E-type perturbation is initially braid-centred perturbations which couple with the billow core and hence trigger an elliptical/translative perturbation there. We believe it is still appropriate to identify this perturbation as of E-type due to the characteristic vorticity distribution shown in figure 16 (analogous to figure 9a), even though its fraction of energy within the core contour ellipse is just below our (frankly arbitrary) criterion, as shown in 

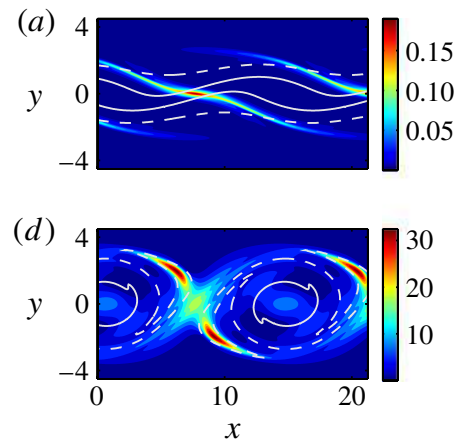

(b)

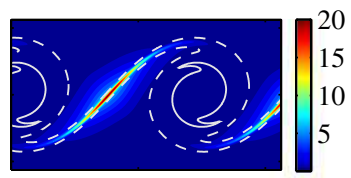

(e)

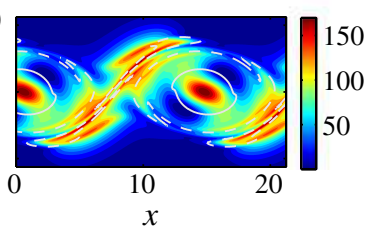

(c)
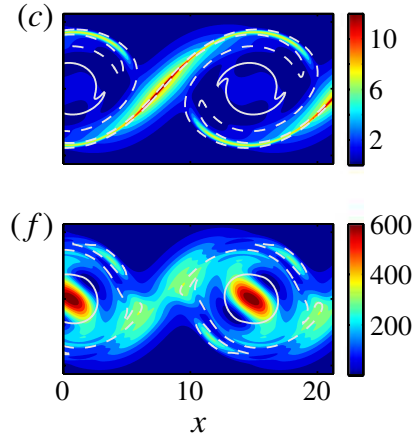

FIGURE 17. Scaled energy density of the optimal perturbation from $t=20$ to $t=60$ with $k_{x}=k_{k h}$ and $k_{z}=\pi / 5$ (E-type) at: $(a) t=20 ;(b) t=35 ;(c) t=40 ;(d) t=45 ;(e) t=55 ;(f)$ $t=60$. At $t=T_{0}=20$, the integral over the domain of the energy density is set to one. Level sets of the instantaneous base flow vorticity at $\omega_{z}=-0.8$ and $\omega_{z}=-0.2$ are plotted with a white solid line and a white dashed line respectively.

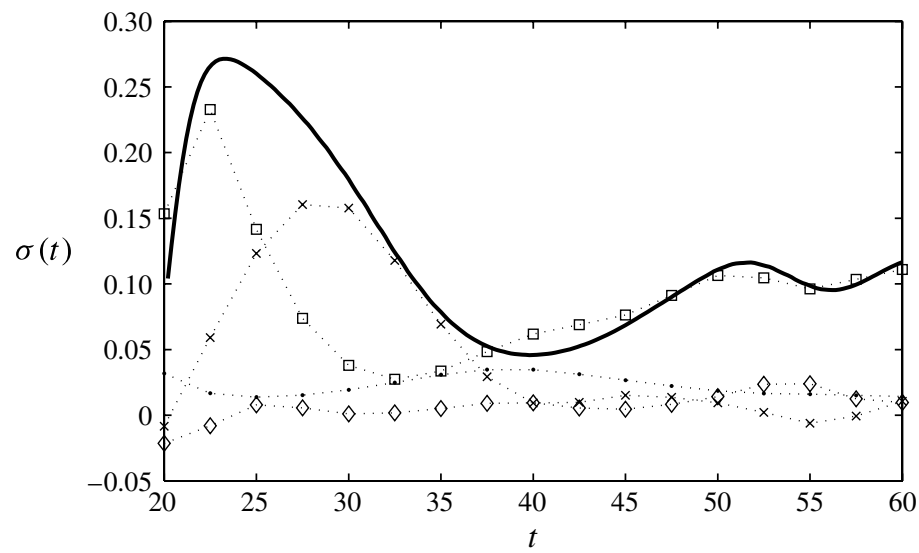

FIGURE 18. Instantaneous growth rate $\sigma(t)$ (thick continuous line) for the optimal perturbation from $t=20$ to $t=60$ and $k_{z}=\pi$ of the H-type mode. The dotted line with $\bullet$ symbols shows the dissipation and also shown are the growth rate contributions from: $\square$, the mean shear; $\diamond$, the shear $2 \mathrm{D}$ and $\times$, the strain $2 \mathrm{D}$.

figure 14. It is clear that once again this relatively low-wavenumber perturbation is strongly dominated by spanwise perturbation vorticity $\omega_{z}$, indicative of the primary billow core perturbation characteristic of the elliptical instability or equivalently E-type perturbation behaviour.

\subsection{H-type response}

On the other hand, removing the early quasi-parallel flow evolution from the optimization interval does not have such a strong effect on the evolution of $\mathrm{H}$ type perturbations, as is apparent from comparison of figures 18, 19 and 20 to their equivalents (for flows with $T_{0}=0$ i.e. figures $7 b, 9 b$ and 11). Considering the instantaneous growth rate first, as before the strain field makes a very strong contribution to the growth of the perturbation after an initial, brief extraction of energy 


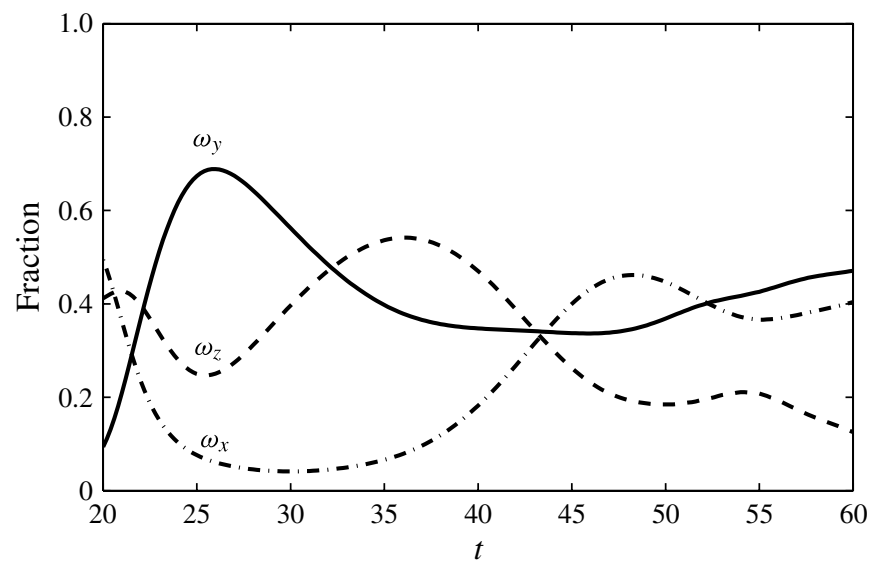

FIGURE 19. Evolution of the fraction of each vorticity component with respect to total enstrophy $\int \omega_{i}(t)^{2} \mathrm{~d} V / \int|\omega(t)|^{2} \mathrm{~d} V$ for the optimal perturbation from $t=20$ to $t=60$ with $k_{x}=k_{k h}$ and $k_{z}=\pi$ (H-type). The continuous line corresponds to $\omega_{y}$, the dashed line corresponds to $\omega_{z}$ and the dash-dotted line corresponds to $\omega_{x}$.
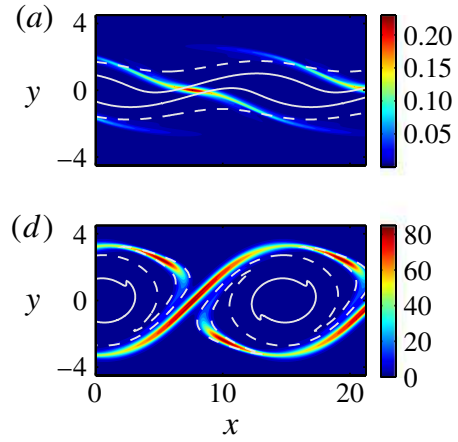

(b)

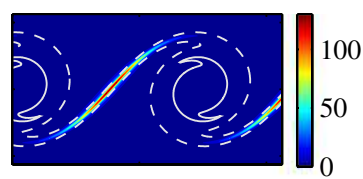

(e)

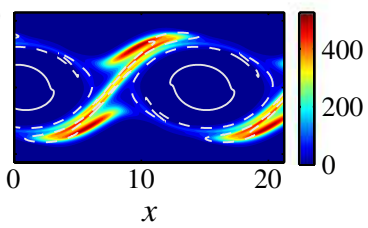

(c)

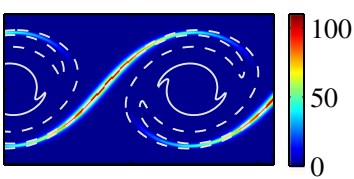

$(f)$

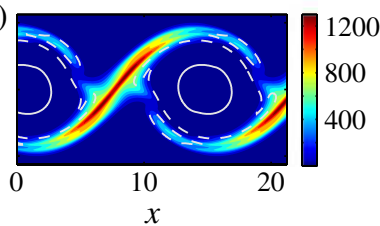

FIGURE 20. Scaled energy density of the optimal perturbation from $t=20$ to $t=60$ with $k_{x}=k_{k h}$ with $k_{x}=k_{k h}$ and $k_{z}=\pi$ (H-type) at: (a) $t=20 ;(b) t=35 ;(c) t=40 ;(d) t=45$; (e) $t=55 ;(f) t=60$. At $t=T_{0}=20$, the integral over the domain of the energy density is set to one. Level sets of the instantaneous base flow vorticity at $\omega_{z}=-0.8$ and $\omega_{z}=-0.2$ are plotted with a white solid line and a white dashed line respectively.

from the mean shear via the Orr/lift-up mechanism. However, as time evolves, the perturbation remains strongly localized in the braid in the vicinity of the hyperbolic fixed point (unlike the E-type perturbation discussed above) and clearly has H-type character. This character is further confirmed by the vorticity compartmentalization shown in figure 19, where the flow is initially dominated once again by vertical (cross-stream) vorticity $\omega_{y}$ which is then stretched and tilted by the braid-centred strain field. Therefore, removing the initial period from the optimization interval appears to have little qualitative effect on the H-type perturbations, and using non-modal stability theory, we appear to be able to identify optimal braid-centred perturbations which are very reminiscent in structure of the previously considered hyperbolic instability. 

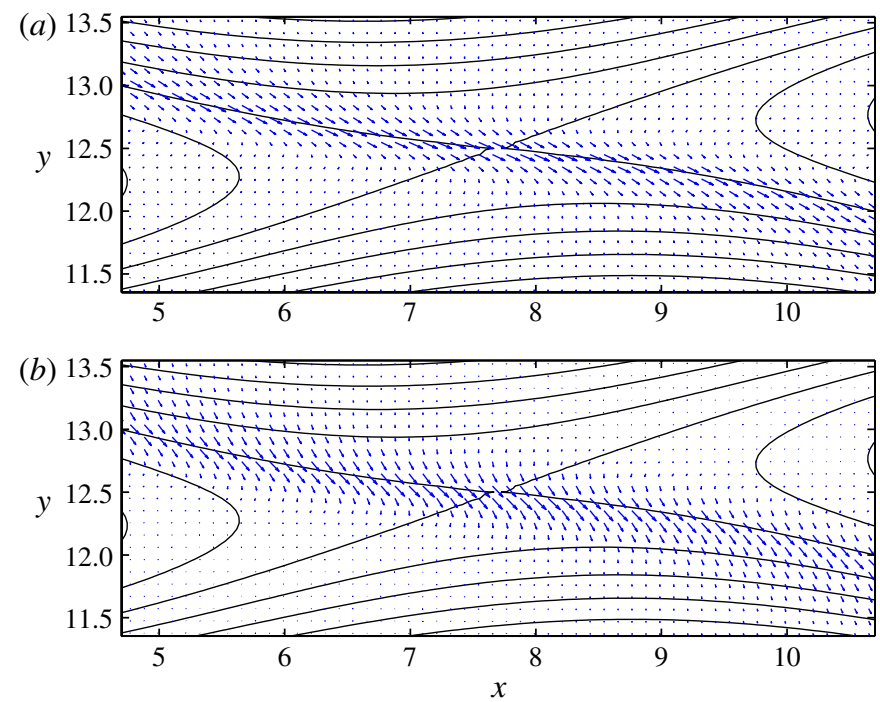

FIGURE 21. (Colour online) Velocity vectors in an $(x, y)$-plane for the initial condition of the optimal perturbations with $k_{z}=\pi / 5$ (E-type) in $(a)$ and $k_{z}=\pi$ (H-type) in $(b)$. The black lines in $(a, b)$ show instantaneous streamlines of the basic flow at the corresponding time $t=20$.

\subsection{Anti-lift-up}

Furthermore, by considering optimal perturbation growth on the flow from the time $T_{0}=20$, the central role of the hyperbolic region in the braid, even for development of ultimately core-centred E-type perturbations can be identified. In figure 21, we plot the velocity vectors in a streamwise plane for the initial time (i.e. at $T_{0}=20$ ) for both the optimal E-type perturbation (figure 21a) and the optimal H-type perturbation (figure $21 b$ ). In both cases, this initial perturbation is concentrated on the contracting manifold of the hyperbolic point in the braid between neighbouring billow cores and predominantly streamwise in the sense that it is locally aligned with the base flow velocity. For both perturbation types, this streamwise perturbation generates streamwise vortices on the instantaneously stretching manifold of the hyperbolic point, leading to a growth mechanism similar to the anti-lift-up mechanism recently discussed in Ortiz \& Chomaz (2011).

This mechanism is associated with a particular combination of convective nonnormality (due to the transport of the perturbation by the base flow from one region to another) and tilting of the base flow vorticity by the perturbation. The difference here is that the cross-stream perturbation velocity is of the same order as the streamwise perturbation velocity, which possibly may be due to the facts that the base flow in the present case has somewhat different structure (with a strong vorticity sheet in the braid region) and is also evolving in time. In the E-type evolution (but not in the H-type evolution characterized by a significantly smaller spanwise wavelength), the spanwise wavelength of the E-type perturbation is sufficiently large for these streamwise vortices, created via the anti-lift-up mechanism, to induce a non-trivial displacement of the primary (evolving) billow core and excite an elliptical type of response of the core which eventually grows. 


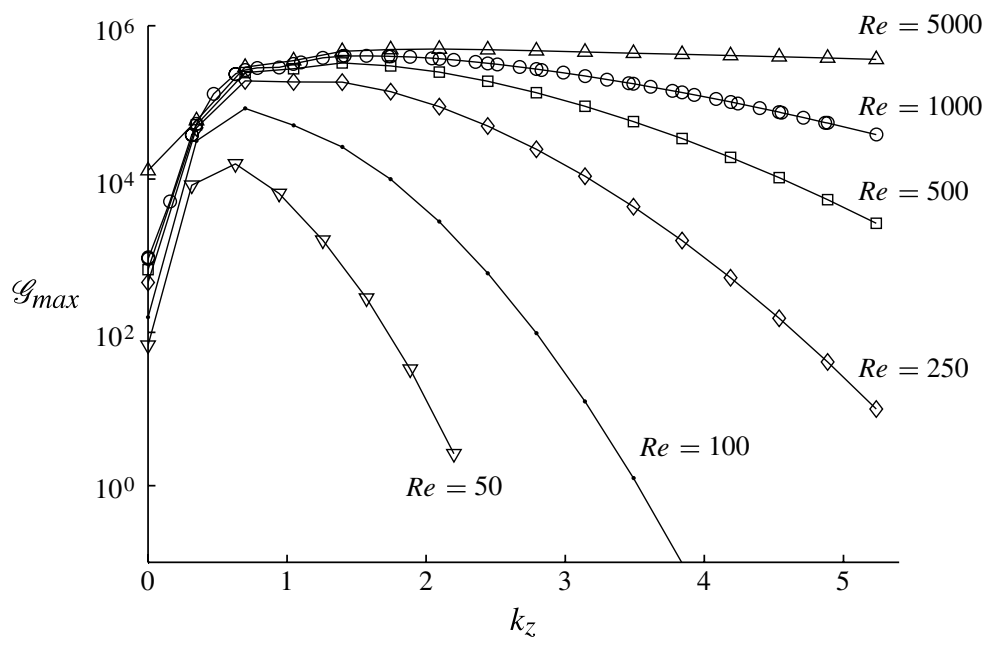

FIGURE 22. Optimal gain versus $k_{z}$ for $T_{0}=20$ and $T=70$ for different Reynolds numbers $R e$, as indicated on the figure.

\subsection{Comparison with other studies with different Re}

As already noted above, Smyth \& Peltier (1994) considered (among other problems) the secondary stability of primary Kelvin-Helmholtz billows, considering in particular the time-dependence of the base flow. The approach which they developed involved the computation of a 'time-averaged stability matrix' (TASM), which essentially corresponded to averaging the base flow during the interval of interest. Most of the results which they presented were for $R e=300$, substantially smaller than considered in this paper, but they also considered the large-Re limit for which they cite evidence of monotonic increase of growth rates with $k_{z}$, which they refer to as 'ultraviolet catastrophe' (Smyth \& Peltier 1994, § 3.2). They performed effectively inviscid computations by using hyperviscosity and showed that the ultraviolet catastrophe appearing with a frozen base flow is suppressed when using the TASM (Smyth \& Peltier 1994, figure 9). They concluded that even a slow time-dependence in the base flow may decorrelate small-scale modes by imposing a short-wave cut-off independent of viscosity.

We revisit this issue here, and we show in figure 22 the gain of optimal perturbations going from $T_{0}=20$ to $T=70$ for various Reynolds numbers between $R e=50$ and $R e=5000$. While for $R e \gtrsim 250$ there is little difference at low $k_{z}$, the optimal gain is strongly dependent on $R e$ for large $k_{z}$. This seems to indicate that it is indeed viscous diffusion that is responsible for the short-wave cut-off observed for smaller Reynolds numbers, and that the relatively slow and small time-dependence of the base flow during this optimization time interval does not of itself preclude a strong instability at small-scales. An interesting aspect of this ultraviolet catastrophe is that it may be related to a large energy transfer directly to the smallest scales, so that it could possibly be related to the mixing transition as described by Dimotakis (2005).

The difference with the TASM-based results of Smyth \& Peltier (1994) might be due to the fact that time-averaging different base flow 'snapshots' has a blurring effect that removes the strong gradients of the base flow, as can be seen below in the inset of figure 23. In essence, the smoothing at the heart of time-averaging approaches such as the TASM method can artificially suppress the significance of small-scale or even 


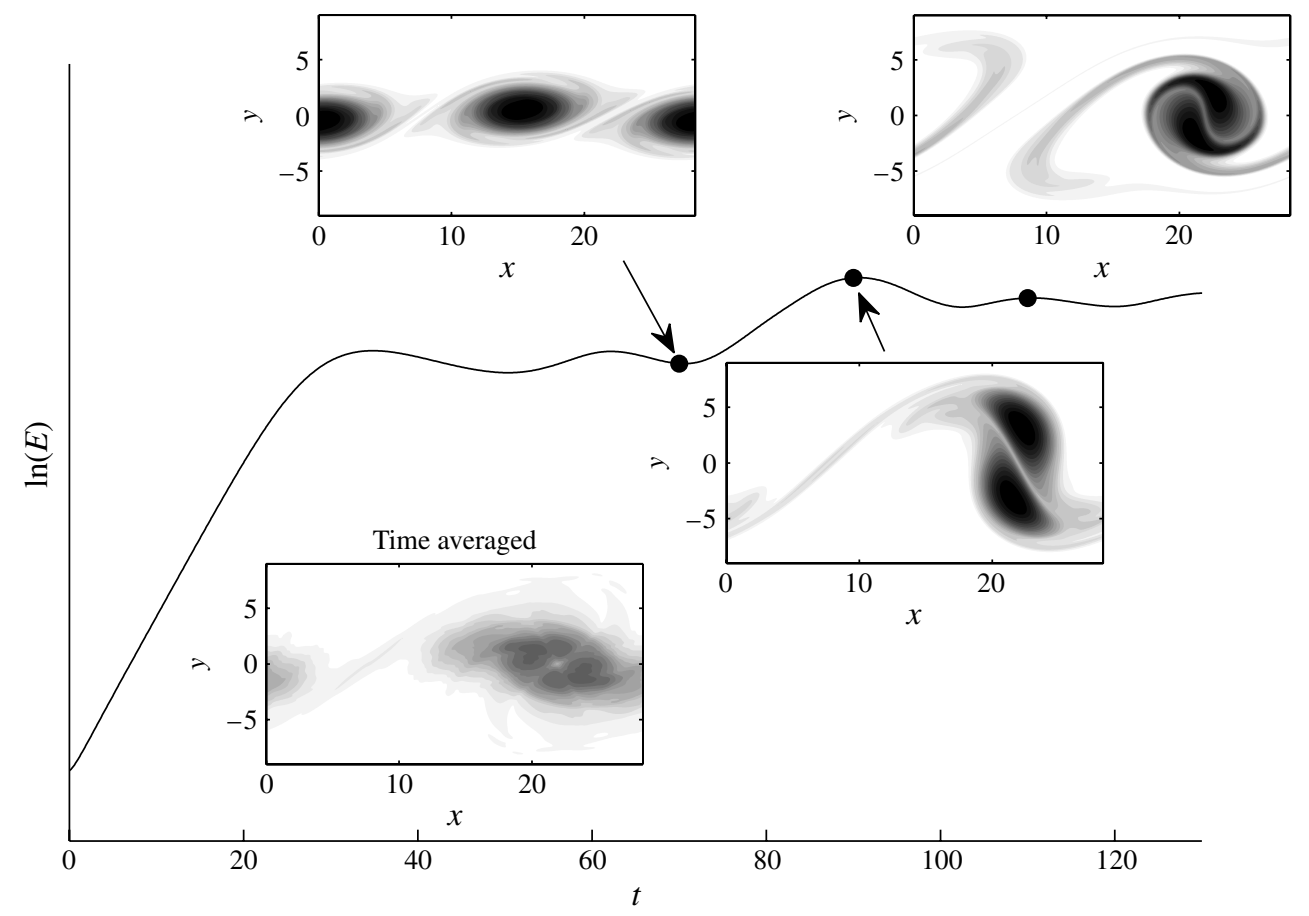

FIGURE 23. Evolution of the pairing base flow. Vertical units are arbitrary. Base flow vorticity is shown from left to right at times $t=70, t=90$ and $t=110$, corresponding to the symbols on the curve. Also shown is the vorticity of the time-averaged base flow; the average is taken from the base flows at $t=70,75,80, \ldots, 110$, which is similar to the pairing-phase base flow used in Smyth \& Peltier (1994) for the stability analysis of the pairing phase. The greyscale is the same in the different vorticity plots, going from white for $\omega_{z}=0$ to black for $\omega_{z}=-1$.

apparently quite minor transient features of the base flow which can play a key role in the growth of secondary perturbations.

Of course, when the transient variation of the base flow is by any measure strong, it is even more necessary to use an algorithm which captures the inherent timedependence of the base state. In the next section, we demonstrate this by considering the effect of the pairing of the primary billows on the optimal transient perturbation identified by our algorithm. We are particularly interested in the effect that pairing has on the E-type and H-type perturbations which we have already identified by consideration of the secondary perturbations which grow on a single primary billow.

\section{Effect of pairing}

\subsection{Pairing base flow}

To consider the effect of pairing on the development of optimal perturbations, we compute another base flow from the same initial condition $\boldsymbol{U}_{b f}\left(T_{0}-\delta t\right)$ used for the previous $T_{0}=0$ base flow (see $\S 4.1$ ). However, in this case, we double the $x$-direction extent, and white noise is added in the region $|y|<5$. The total energy of the added white noise is $5.1464 \times 10^{-4}$, corresponding to $\sim 60 \%$ of the energy of the eigenmode before the noise addition. This noise is added to project at least some energy onto 


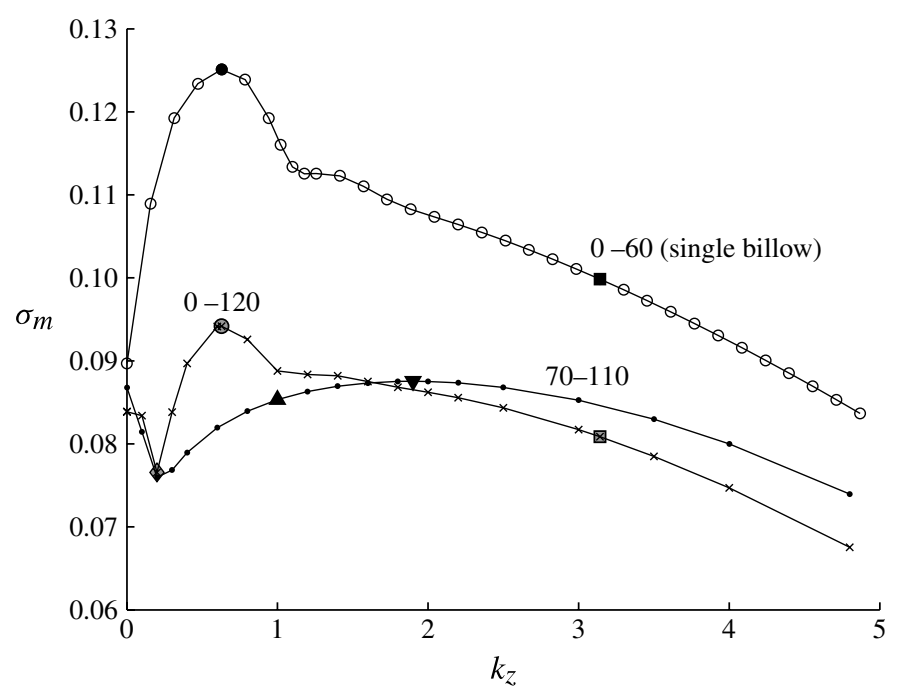

FIGURE 24. Mean optimal growth rate versus $k_{z}$ for different base flows and optimization times: open circles denote the curve for a single-billow base flow for optimization interval $[0,60]$ as also shown in figure 5, where the and symbols on the $T=60$ curve correspond respectively to the E-type and H-type perturbations described in more detail in $\S 4$; crosses denote the curve for a double-billow base flow for optimization interval $[0,120]$ (including pairing), where the grey circle and grey square denote the perturbations with the same wavenumber as the one-billow E-type and H-type perturbations described in more detail below, while the grey diamond denotes the small $\left(k_{z}=0.2\right)$ 'bulging' perturbation discussed in more detail below; dots denote the curve for a double-billow base flow for optimization interval centred on the pairing [70,110], where the up and down triangles denote the perturbations discussed in more detail and compared with the $k_{z}=1$ and $k_{z}=1.9$ perturbations discussed in Smyth \& Peltier (1994).

the well-known subharmonic secondary instabilities of arrays of primary billows, as discussed in detail in Klaassen \& Peltier (1989).

We show aspects of the evolution of this base flow in figure 23. Vorticity snapshots around the time when the primary billows pair are shown at times $t=70,90$ and 110 , which are to be compared with the base flow shown in figure 1 in Smyth \& Peltier (1994), in particular at their times $t=60,80$ and 100. It is important to appreciate that with this particular intensity of forcing, pairing starts roughly at the time $(T=70)$ which we consider as the end of our optimization intervals in the previous sections. It is also clear that pairing is both a relatively quick and very significant perturbation of the base flow.

We show the mean optimal growth rate $\sigma_{m}$ for perturbations to the pairing base flow in figure 24 for $T_{0}=0$ and $T=120$, and for $T_{0}=70$ and $T=110$ (analogous to the pairing-phase computations of Smyth \& Peltier 1994). For comparison we also show the corresponding $\sigma_{m}$ for a single billow and optimization from $T_{0}=0$ to $T=60$, whose corresponding optimal gain has already been shown in figure 5. The $\sigma_{m}$ of the optimal perturbations in the pairing phase (from 70 to 110 , curve with black dots in figure 24) is substantially larger than the growth rates of the unstable eigenmodes found by Smyth \& Peltier (1994) (see their figure 5) using the TASM method in the corresponding interval. 


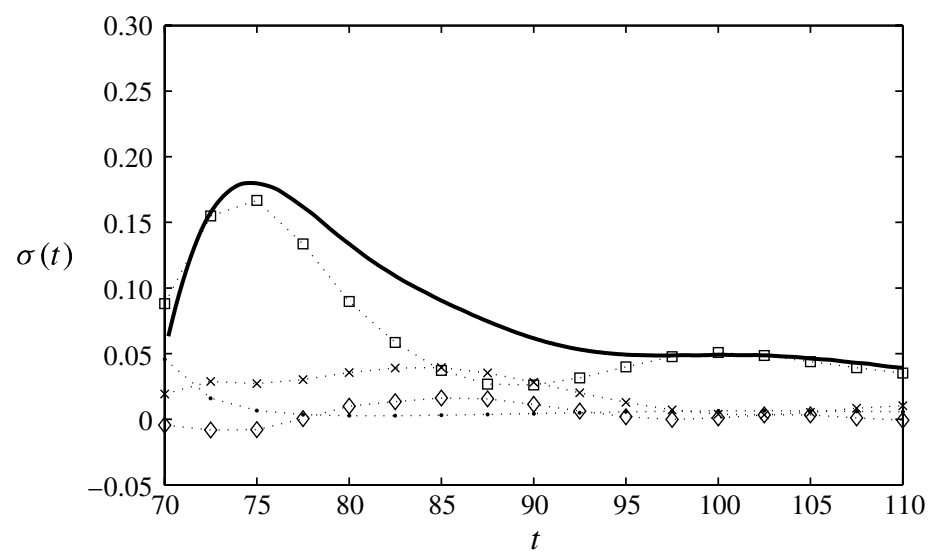

FIGURE 25. Instantaneous growth rate $\sigma(t)$ (thick continuous line, as defined in (3.7)), for the optimal perturbations from $t=70$ to $t=110$ with $k_{z}=1$. The dotted line with $\bullet$ symbols shows the dissipation and also shown are the growth rate contributions from: $\square$, the mean shear; $\diamond$, the shear $2 \mathrm{D}$ and $\times$, the strain $2 \mathrm{D}$.

A more qualitative difference between the present results and the TASM results of Smyth \& Peltier (1994) can be observed for $k_{z} \lesssim 1$, for which their results present an abrupt change in the slope, probably due to the presence of core-centred modes, related to the E-type perturbations discussed previously. This is largely unsurprising, as the time-averaged flow still retains a noticeable elliptical vortex (from the merged billows), while the time-dependent flow does not, as can be seen by comparing the different base flows in figure 23.

\subsection{Optimal perturbations for $T_{0}=70, T=110$ during the pairing phase}

For comparison with the results of Smyth \& Peltier (1994), we identify the optimal perturbations on a pairing base flow where the optimization interval is chosen to correspond with the period when the pairing occurs, i.e. $T_{0}=70$ and $T=110$. During this period, the base flow is changing rapidly and significantly, and so the inherent time-dependence of our approach is well-suited to this calculation. For $k_{z}=1$ (marked with an upward filled triangle on figure 24) Smyth \& Peltier (1994) computed the optimal perturbation during the pairing phase using the time-averaged flow as the base flow. They report a value of $\sigma_{m}=0.0731$ with their approximate method, somewhat smaller than but still surprisingly close to our result $\sigma_{m}=0.08533$.

We show the time evolution of the instantaneous growth rate and the various energetic contributions in figure 25 , the various vorticity components in figure 26 , and various snapshots of the energy density in figure 27 . We also show the equivalent figures for the most amplified of the computed optimal perturbations, which occurred for $k_{z}=1.9$ (marked with a downward filled triangle on figure 24) with only a slightly larger mean growth rate of $\sigma_{m}=0.08754$ in figures 28, 29 and 30 respectively. By comparison with the equivalent figures for the single-billow flow optimized over $[0,60]$ discussed in $\$ 4$ (i.e. figures 7,9 and 11) it is apparent that these perturbations, even at the smaller $k_{z}=1$ wavenumber, have the central characteristics of braidcentred H-type perturbations, and really have little in common with the core-centred E-type perturbations which develop at lower wavenumbers on a single-billow base flow. 


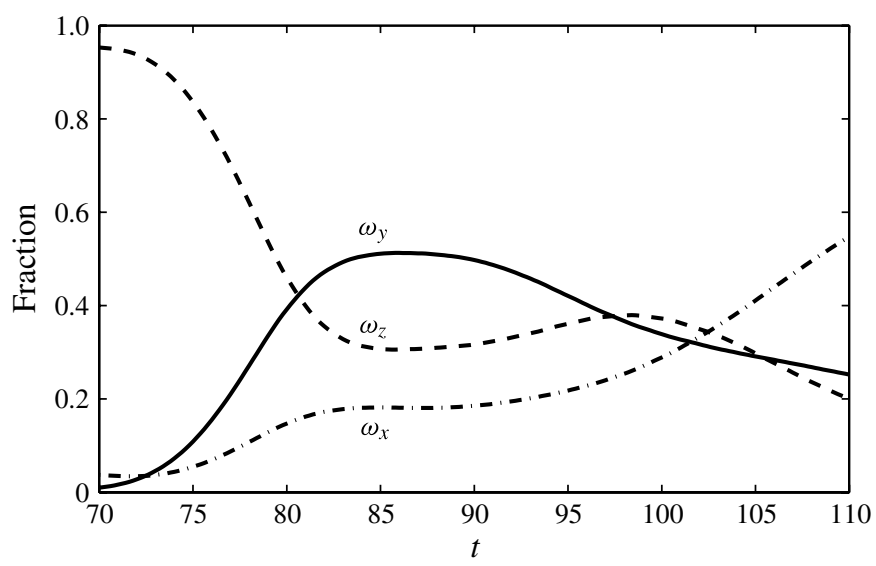

FIGURE 26. Evolution of the fraction of each vorticity component with respect to total enstrophy $\int \omega_{i}(t)^{2} \mathrm{~d} V / \int|\omega(t)|^{2} \mathrm{~d} V$ for the optimal perturbation from $t=70$ to $t=110$ with $k_{z}=1$. The continuous line corresponds to $\omega_{y}$, the dashed line corresponds to $\omega_{z}$ and the dash-dotted line corresponds to $\omega_{x}$.

(a)

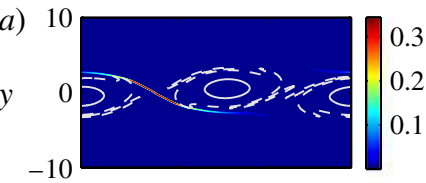

(d)

$d)$
$y$

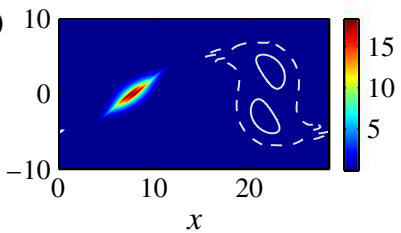

(b)

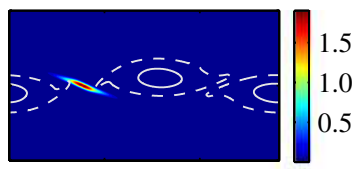

(e)

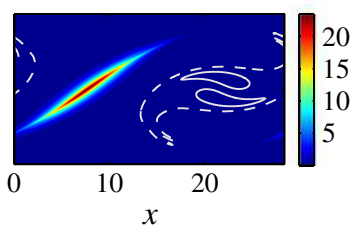

(c)

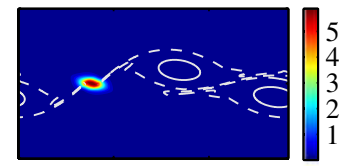

$(f)$

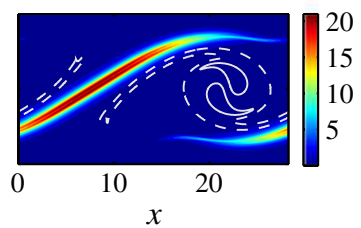

FIGURE 27. Scaled energy density of the optimal perturbation during the pairing phase from $t=70$ to $t=110$ with $k_{z}=1$ at: $(a) t=70 ;(b) t=75 ;(c) t=80 ;(d) t=90 ;(e) t=100 ;(f)$ $t=110$. At $t=T_{0}=70$, the integral over the domain of the energy density is set to one. Level sets of the instantaneous base flow vorticity at $\omega_{z}=-0.8$ and $\omega_{z}=-0.2$ are plotted with a white solid line and a white dashed line respectively.

This identification as an H-type perturbation is particularly clear from the dominance of the vertical vorticity $\omega_{y}$ and from the structure of the scaled energy density. The optimal perturbations rapidly become strongly localized in the braid region which remains between neighbouring (due to the imposed periodicity) pairing billows, which is located around $x=10$ in the figures. Due to the intensity of the pairing process, this surviving braid region allows substantial energy growth due to inherently non-parallel 2D shear and strain (plotted with diamonds and crosses respectively in figures 25 and 28) during the inherently time-dependent variation of the base flow as the pairing process occurs.

Conversely, due to the complete disruption of the primary billow cores, the growth of core-centred E-type perturbations appears to be strongly suppressed, and so during pairing, secondary instabilities appear to avoid in some sense the primary billow cores. 


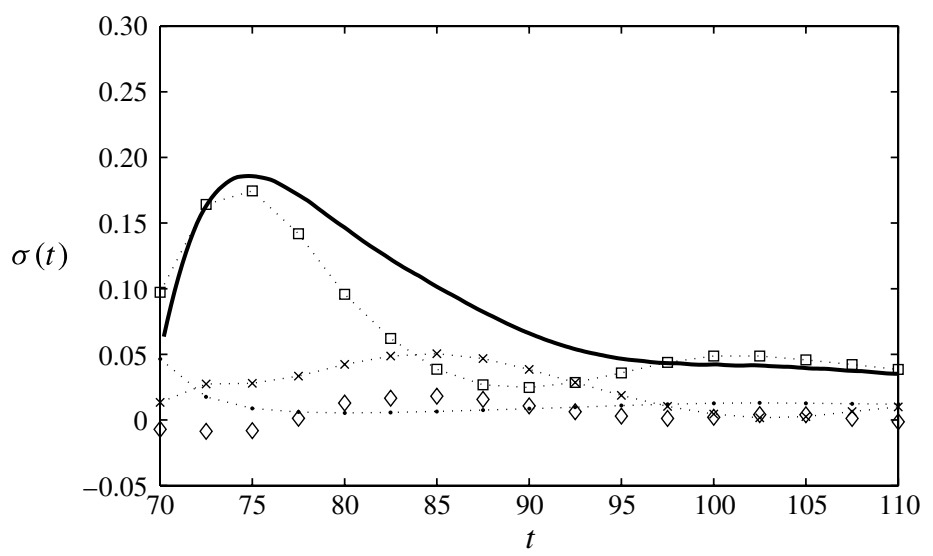

FIGURE 28. Instantaneous growth rate $\sigma(t)$ (thick continuous line) for the optimal perturbation on the pairing phase from $t=70$ to $t=110$ and $k_{z}=1.9$. The dotted line with - symbols shows the dissipation and also shown are the growth rate contributions from: $\square$, the mean shear; $\diamond$, the shear $2 \mathrm{D}$ and $\times$, the strain $2 \mathrm{D}$.

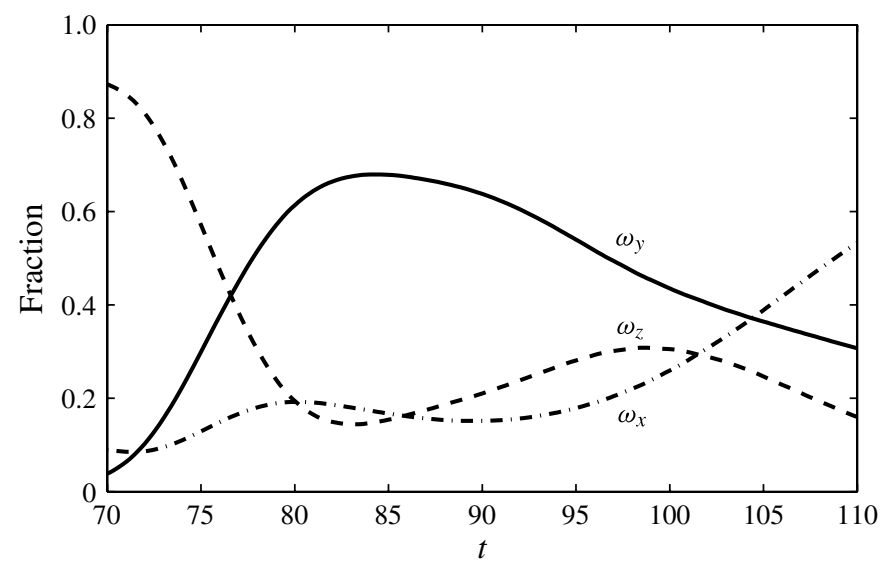

FIGURE 29. Evolution of the fraction of each vorticity component with respect to total enstrophy $\int \omega_{i}(t)^{2} \mathrm{~d} V / \int|\omega(t)|^{2} \mathrm{~d} V$ for the optimal perturbation from $t=70$ to $t=110$ with $k_{z}=1.9$. The continuous line corresponds to $\omega_{y}$, the dashed line corresponds to $\omega_{z}$ and the dash-dotted line corresponds to $\omega_{x}$.

This picture is qualitatively different in structure from that identified by Smyth \& Peltier (1994) (cf. their figure 7) where the TASM method identified the optimal perturbation with wavenumber $k_{z} \simeq 1$ as having perturbation kinetic energy initially in the core and in the region between the braid and the core at the final time. From the time-averaged base flow shown in figure 23, this is not entirely surprising, as the averaged base flow approximates the base flow as a single billow. However, our calculations demonstrate the critical role that time-dependence of the base flow plays in driving the secondary perturbation growth. 

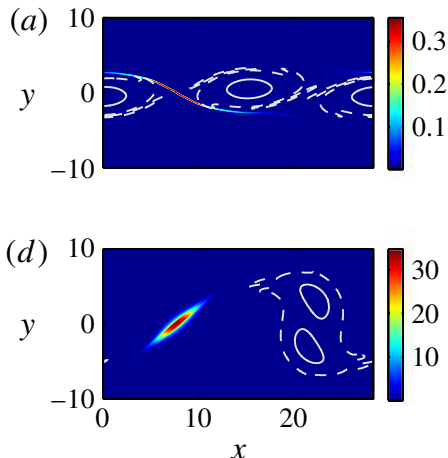

(b)

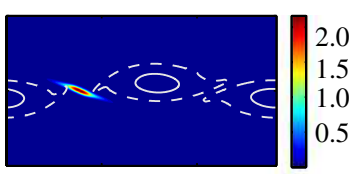

(e)

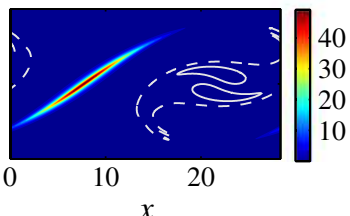

(c)

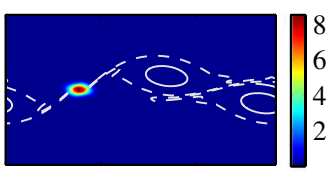

$(f)$

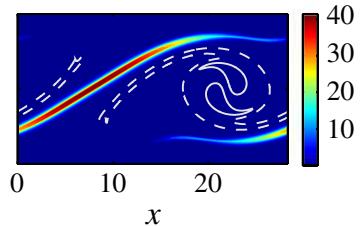

FIGURE 30. Scaled energy density of the optimal perturbation during the pairing phase from $t=70$ to $t=110$ with $k_{z}=1.9$ at: $(a) t=70 ;(b) t=75 ;(c) t=80 ;(d) t=90 ;(e) t=100 ;(f)$ $t=110$. At $t=T_{0}=70$, the integral over the domain of the energy density is set to one. Level sets of the instantaneous base flow vorticity at $\omega_{z}=-0.8$ and $\omega_{z}=-0.2$ are plotted with a white solid line and a white dashed line respectively.

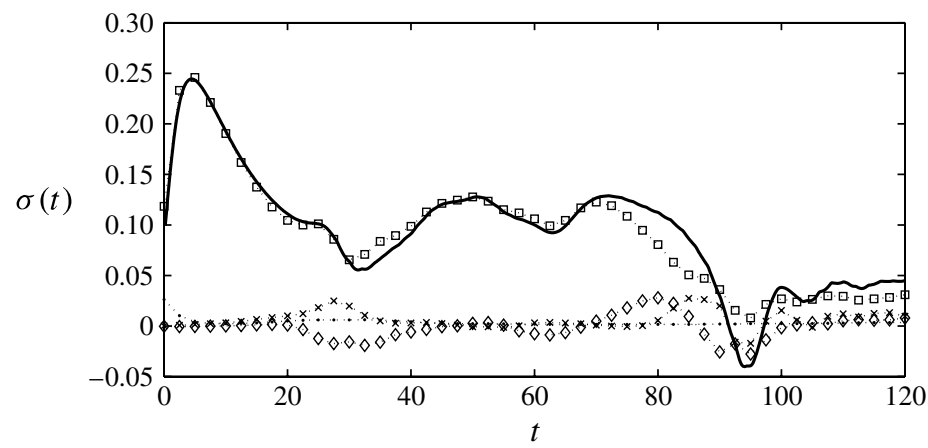

FIGURE 31. Instantaneous growth rate $\sigma(t)$ (thick continuous line) for the optimal perturbation from $t=0$ to $t=120$ and $k_{z}=\pi / 5$ (E-type). The dotted line with $\bullet$ symbols shows the dissipation and also shown are the growth rate contributions from: $\square$, the mean shear; $\diamond$, the shear $2 \mathrm{D}$ and $\times$, the strain $2 \mathrm{D}$.

\subsection{Optimal perturbations for $T_{0}=0, T=120$ with pairing}

However, if the optimization interval extends over the whole evolution of the base flow from $T=0$, it is still possible for E-type perturbations to grow substantially, essentially because they are able to grow and become established on the individual primary billows before they start to pair, although the average growth rate of the perturbations is markedly reduced from that which occurs on a single-billow base flow, as is apparent in figure 24. For the E-type perturbation $\left(k_{z}=\pi / 5\right)$ optimized from $t=0$ to $t=120$, We show the time evolution of the instantaneous growth rate and the various energetic contributions in figure 31, the various vorticity components in figure 32, and various snapshots of the energy density in figure 33. By comparison with the equivalent figures for the single-billow flow optimized over $[0,60]$ discussed in $\$ 4$ (i.e. figures $7 a, 8$ and $9 a$ ) it is apparent that these perturbations have the central characteristics of core-centred E-type perturbations, and the initial evolution is quantitatively very similar to that of the previously discussed core-centred E-type 


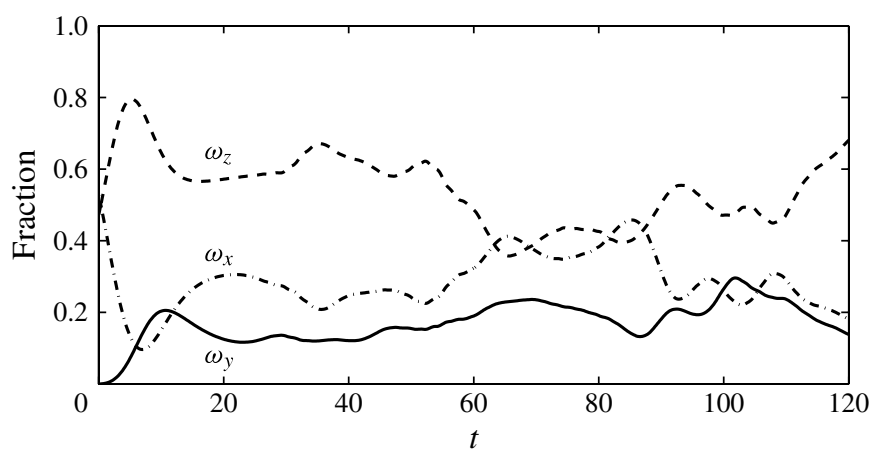

FIGURE 32. Evolution of the fraction of each vorticity component with respect to total enstrophy $\int \omega_{i}(t)^{2} \mathrm{~d} V / \int|\omega(t)|^{2} \mathrm{~d} V$ for the optimal perturbation from $t=0$ to $t=120$ with $k_{z}=\pi / 5$ (E-type). The continuous line corresponds to $\omega_{y}$, the dashed line corresponds to $\omega_{z}$ and the dash-dotted line corresponds to $\omega_{x}$.
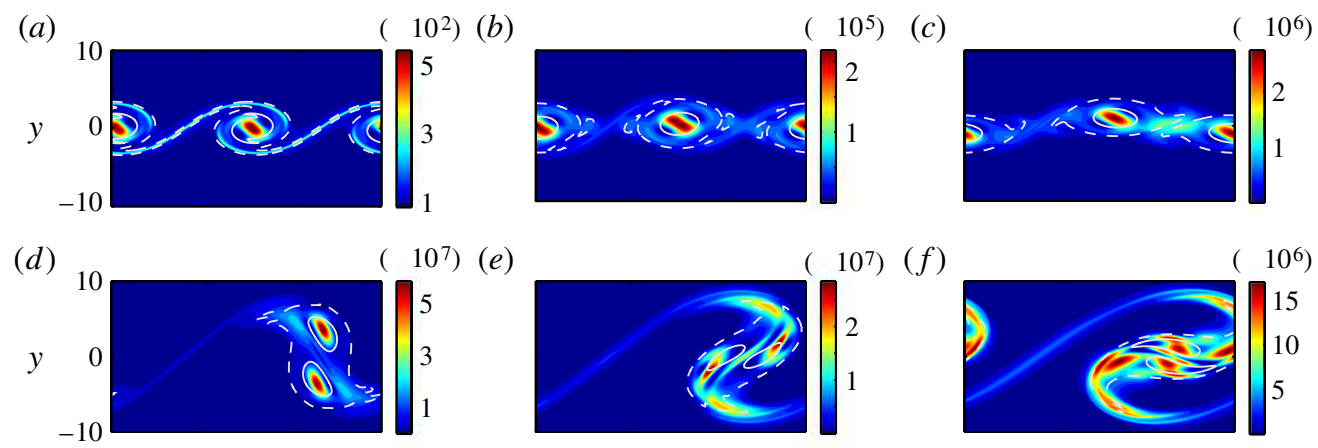

$(e)$
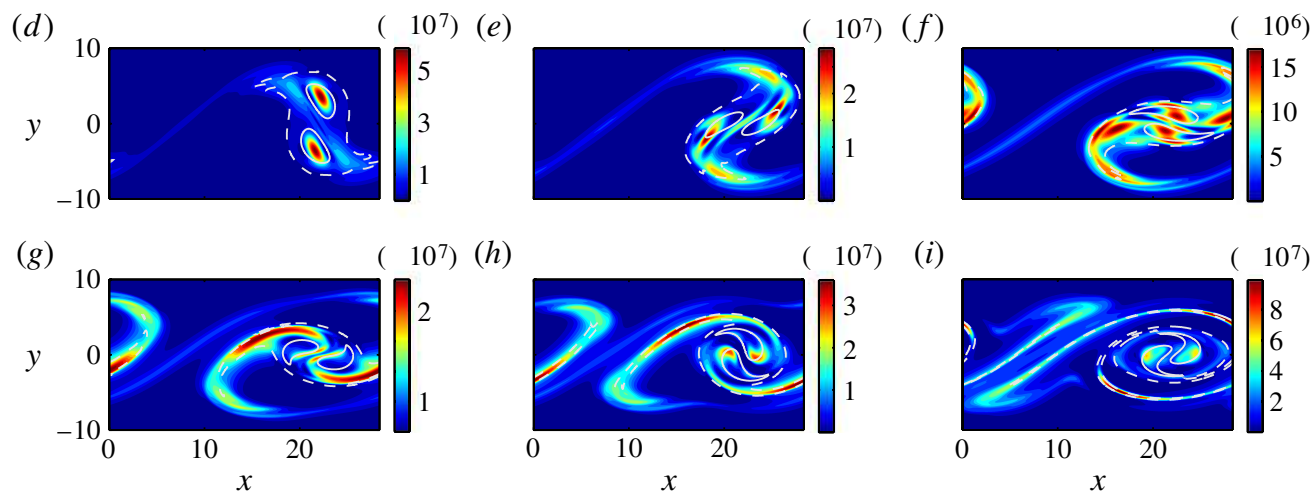

(i) $\left(\times 10^{7}\right)$

FIGURE 33. Scaled energy density of the optimal perturbation including the pairing phase from $t=0$ to $t=120$ with $k_{z}=\pi / 5$ (E-type) at: (a) $t=35$; (b) $t=65$; (c) $t=75$; (d) $t=90$; (e) $t=95 ;(f) t=100 ;(g) t=105$; $(h) t=110$; ( $i) t=120$. At $t=T_{0}=0$, the integral over the domain of the energy density is set to one. Level sets of the instantaneous base flow vorticity at $\omega_{z}=-0.8$ and $\omega_{z}=-0.2$ are plotted with a white solid line and a white dashed line respectively.

perturbations on a single-billow base flow. During the pairing phase, the growth of the perturbation is strongly suppressed, but the perturbation still manages to survive as an essentially core-centred perturbation.

A very similar picture emerges for the H-type perturbation $\left(k_{z}=\pi\right)$ optimized from $t=0$ to $t=120$, for which we plot the energy growth rate and the various contributions in figure 34 , the vorticity components in figure 35, and various snapshots 


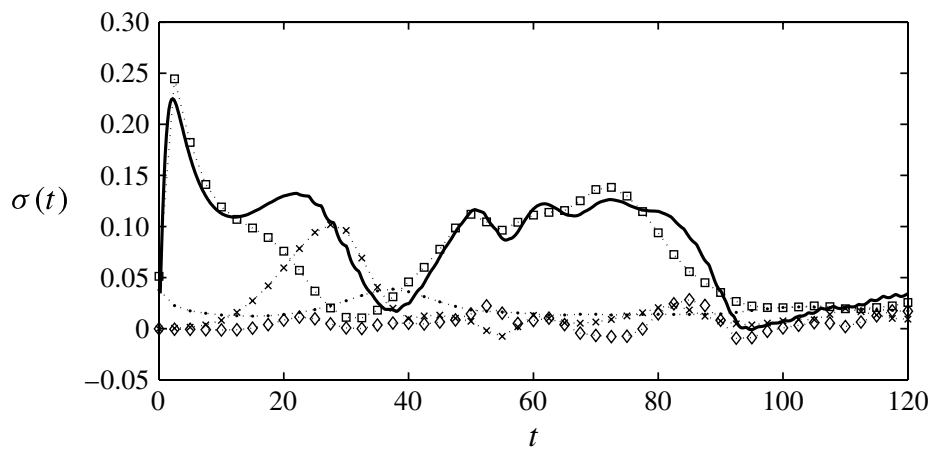

FIGURE 34. Instantaneous growth rate $\sigma(t)$ (thick continuous line) for the optimal perturbation from $t=0$ to $t=120$ and $k_{z}=\pi$ (H-type). The dotted line with $\bullet$ symbols shows the dissipation and also shown are the growth rate contributions from: $\square$, the mean shear; $\diamond$, the shear $2 \mathrm{D}$ and $\times$, the strain $2 \mathrm{D}$.

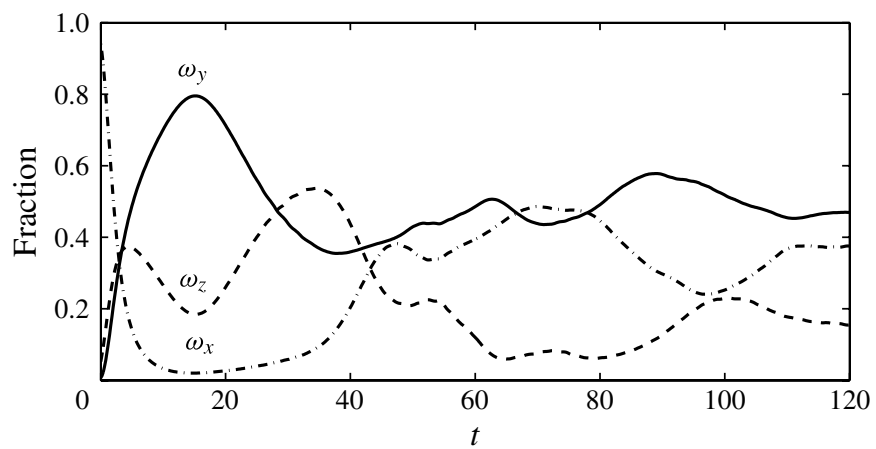

FIGURE 35. Evolution of the fraction of each vorticity component with respect to total enstrophy $\int \omega_{i}(t)^{2} \mathrm{~d} V / \int|\omega(t)|^{2} \mathrm{~d} V$ for the optimal perturbation from $t=0$ to $t=120$ with $k_{z}=\pi$ (H-type). The continuous line corresponds to $\omega_{y}$, the dashed line corresponds to $\omega_{z}$ and the dash-dotted line corresponds to $\omega_{x}$.

of the energy density in figure 36. Once again, the initial evolution is quantitatively similar to the evolution of the optimal H-type perturbation with the same wavenumber which develops on a single-billow base flow over the optimization interval $[0,60]$ discussed in $\S 4$, as shown in figures $7(b), 9(b)$ and 11. As with the optimal perturbations for the optimization interval centred on the pairing phase discussed in $\S 6.2$, the optimal perturbation selects the braid which survives during the pairing of the primary billows, and thus is able to grow in the braid until the pairing of the primary billows is quite well-advanced. The time-dependent evolution of this secondary optimal perturbation can be followed over the pairing phase of course, and it is clear that the identification of both the E-type and the H-type perturbations as being optimal is robust for pairing as well as single billows.

Finally, it is also possible to consider small (spanwise) wavenumber optimal perturbations $\left(k_{z}=0.2\right.$, as marked with a diamond on figure 24) as a possible candidate for a bulging mode of the paired billows, as previously discussed by Rogers \& Moser (1992) and Schoppa, Hussain \& Metcalfe (1995) among others. 
(a)

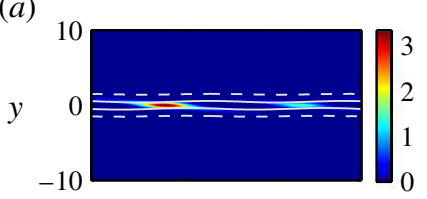

(d)

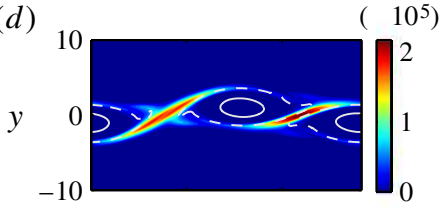

(g)

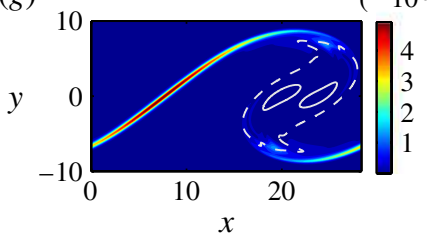

(b)

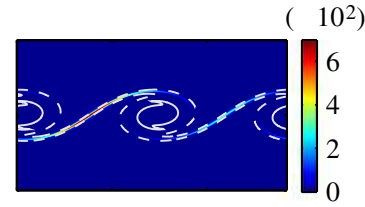

$\left(\times 10^{5}\right)(f)$

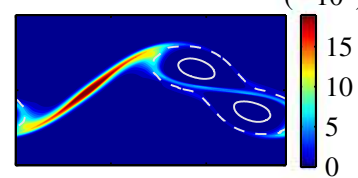

$\left(\times 10^{6}\right)$

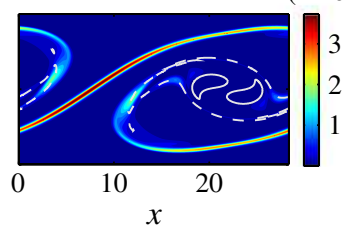

(c)

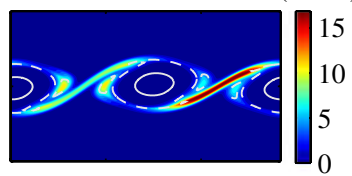

$(f)$

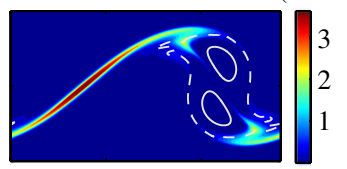

(i)

$\left(\times 10^{6}\right)$

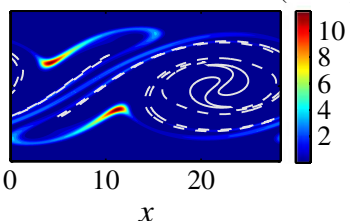

FIGURE 36. Scaled energy density of the optimal perturbation including the pairing phase from $t=0$ to $t=120$ with $k_{z}=\pi$ (H-type) at: (a) $t=10 ;(b) t=35 ;(c) t=65 ;(d) t=75$; (e) $t=85$; (f) $t=90$; ( $g$ ) $t=95$; (h) $t=105$; ( $i) t=120$. At $t=T_{0}=0$, the integral over the domain of the energy density is set to one. Level sets of the instantaneous base flow vorticity at $\omega_{z}=-0.8$ and $\omega_{z}=-0.2$ are plotted with a white solid line and a white dashed line respectively.

Consideration of the instantaneous growth rate, the various energy contributions, the components of the vorticity and the spatial distribution of the energy density all suggest that this perturbation has many aspects in common with an E-type perturbation, being largely core-centred (after a brief braid-centred initial transient) and dominated by spanwise $\omega_{z}$ vorticity perturbations. However, important differences can be appreciated at the end of the optimization interval at $T=120$, as shown in figure 37, indicating that the low-wavenumber optimal perturbation leads to the characteristic features of the 'core dynamics instability' of Schoppa et al. (1995). In particular, the undulations of the isocontours $\omega_{z}=-0.6$ and $\omega_{z}=-0.9$ are in phase and produce a modulation of the core thickness, which corresponds to the bulging of the core, a feature different from the E-type for which the inner and outer deformations of the core are out of phase and correspond to a translation of the core with no evident thickness modulation (figure 10). Consistently, the sign of $\omega_{x}$ is different at the two sides of the core for the bulging instability of figure 37, in contrast with the E-type perturbation shown previously in figure 10. In summary therefore, it appears that the direct-adjoint looping technique which we use is admirably suited to the identification of optimal perturbations in mixing layers, even when the primary billows are allowed to pair.

\section{Conclusions}

In this paper, we have investigated numerically the transient linear growth of 3D perturbations in an homogeneous time-evolving shear layer, which are optimal in terms of their kinetic energy gain over a finite, predetermined time interval. We use the classic direct-adjoint looping method combined with power iteration to identify 


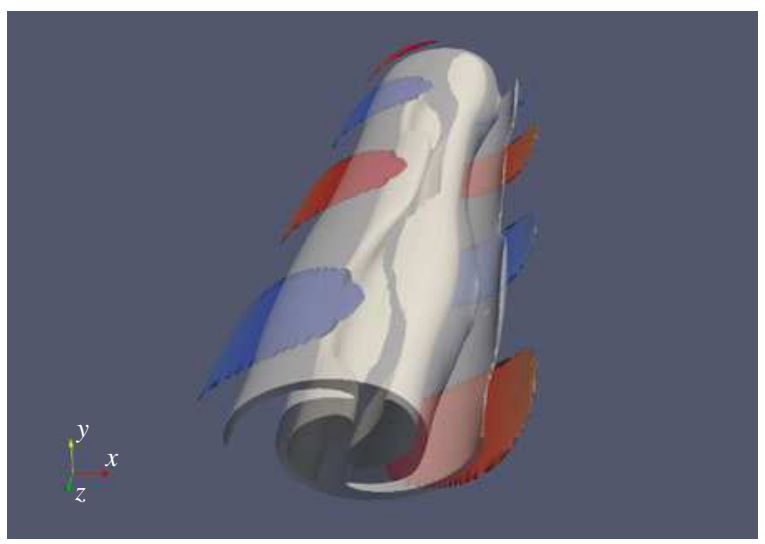

FIGURE 37. Optimal perturbation with $k_{z}=0.2$ at time $t=120$ superposed on the base flow. The perturbation amplitude is such that the maximum value of the $\omega_{z}$ vorticity (spanwise) is 0.4 . The white semi-transparent contour corresponds to the isocontour $\omega_{z}=-0.6$ and the solid white isocontour corresponds to $\omega_{z}=-0.9$. The red/blue contours correspond to $\left(\omega_{x}^{2}+\omega_{y}^{2}\right)^{1 / 2}=0.2$, the colouring being according to the magnitude of $\omega_{x}$, red (respectively blue) representing positive (negative) values of $\omega_{x}$.

the optimal perturbations. We model the shear layer with an initial parallel velocity distribution $\boldsymbol{U}(y)=U_{0} \tanh (y / d) \boldsymbol{e}_{x}$ with Reynolds number $\operatorname{Re}=U_{0} d / v=1000$, where $v$ is the kinematic viscosity of the fluid. To understand whether the primary KH instability is essential to the transient perturbation growth, we have considered three different situations in detail. By use of a fictitious body force to counteract the effect of viscosity on the base flow shear layer, we are able to consider the transient finite-Re perturbation growth in isolation of the primary $\mathrm{KH}$ billow growing. We then consider the transient growth, right from the start $\left(T_{0}=0\right)$ of the roll-up of the primary billow, which initially involves a relatively short period when the time-evolving base flow is actually quite close to the (strictly) parallel frozen base flow. Finally, we consider an optimization from $T_{0}=20$ (the primary billow saturates at $t_{\text {sat }}=35$ ) which essentially means that the base flow is strongly non-parallel throughout the optimization interval.

For short enough times, the most amplified perturbations on the frozen tanh profile are inherently 3D, and are most appropriately described as hybrid or mixed perturbations which grow through a subtle combination of the lift-up and Orr mechanisms (OL-type), while for longer times, the optimal perturbations are 2D, and are very similar to the $\mathrm{KH}$ normal mode, with a slight enhancement of gain due to extraction through the Orr mechanism (which we thus refer to as a Ktype perturbation). However, for the two illustrative cases where we consider the time-evolving $\mathrm{KH}$ flow, we find that the structure of the predicted linear optimal perturbations depends crucially on the length and starting value of the optimizing time interval, as well as the particular spanwise wavenumber selected. If the time-evolving problem has an optimization interval which involves a base flow close in some sense to the initial parallel hyperbolic-tangent shear flow for some non-trivial period, we find that hybrid OL-type perturbations continue to dominate over sufficiently short time intervals.

For longer time intervals however, which involve substantial evolution of the nonparallel primary $\mathrm{KH}$ billow into isolated elliptical vortices, we find two broad classes 
of inherently 3D linear optimal perturbations, associated with different spanwise wavenumber. These two classes have clear points of similarity at low wavenumbers with the well-known core-centred instability identified by Pierrehumbert \& Widnall (1982) and described as elliptical and explained physically by Waleffe (1990), and at higher wavenumbers with the braid-centred hyperbolic instability first reported by Klaassen \& Peltier (1991). Therefore, we refer to these as E-type and H-type perturbations respectively, as they have signatures associated with spanwise and vertical perturbation vorticity respectively. We find that the H-type perturbation (at high wavenumber of the same order as that observed numerically by Caulfield \& Peltier 2000) is relatively inefficient in extracting gain via the Orr and lift-up mechanisms.

However, the smaller-wavenumber core-centred E-type perturbations are very efficient initially at extracting energy from the base flow by the Orr and lift-up mechanisms. Indeed, the flow evolution of such core-centred modes seems to pass through two stages. Initially OL-type behaviour occurs. Since in the time-evolving flow there is some structural differentiation between the braid and the core, this early-time OL-type behaviour is actually most strong in the braid. A spanwise-periodic structure develops there which then strongly perturbs the billow core, causing it to 'ring' and thus triggers vigorous E-type behaviour. When the start time of the optimization interval does not allow the OL-type hybrid perturbations much opportunity to grow, the dominant gain of the E-type perturbations is suppressed relative to the braidcentred H-type perturbations, essentially because the OL-type perturbation cannot grow so vigorously to trigger the core-centred E-type behaviour.

Nevertheless, we have demonstrated that non-modal stability analysis naturally recovers E-type and H-type perturbations in a time-evolving shear layer flow with qualitatively different growth mechanisms and spatial localizations. This implies that both types of secondary instabilities can develop essentially independently. Since the relative significance of the $\mathrm{H}$-type perturbations appears to increase as the start time $T_{0}$ of the optimization interval increases from zero, our results may be interpreted to suggest that the observed dominance of relatively high-wavenumber braid-centred rib vortices is associated with the secondary perturbations only starting to develop some time after the initial $\mathrm{KH}$ quasi-2D instability starts to grow. On the other hand, since E-type perturbations can be greatly influenced by perturbations at the very early, nearly parallel stages of the flow development, our results may be thought of as being related to the generation of distinct vorticity patterns by periodic modulations of the splitter plate at the origin of the mixing layer, as discussed in Lasheras \& Choi (1988). Furthermore, we have found that both E-type and H-type perturbations can grow significantly, even when the primary $\mathrm{KH}$ instabilities pair, provided the optimization interval is sufficiently long to allow the development of the perturbations on the primary billows before these billows start to interact with each other. This initial stage of perturbation growth is particularly important for E-type perturbations, as we have shown that if the optimization interval is restricted to consider only the pairing phase E-type perturbations are strongly suppressed, as they inherently appear to require the primary billow structure to be at most slowly varying to grow, centred as they are on the primary billow cores.

However, the relevance of these linear calculations to such nonlinear flow evolution (and transition mechanisms) as the actual development of rib vortices, or indeed bulging of a larger vortex arising from a pairing event is of course not yet established. In particular, the observed dominance of rib vortices may well be due to such structures being more well-suited to finite-amplitude nonlinear energy extraction from 
the base flow, for example by nonlinear vortex stretching by the strain flow in the vicinity of the hyperbolic point in the braid. Further work is required involving careful direct nonlinear simulation to determine whether these non-modal stability calculations have identified realistic and relevant routes to transition. A particularly interesting question which could be investigated is whether OL-type perturbations, at sufficiently high amplitude could completely preclude the roll-up of K-type $\mathrm{KH}$ billows, and thus, in some sense lead to bypass transition. Alternatively, it would be very interesting to follow into the nonlinear regime the competition between E-type and H-type perturbations, or indeed their nonlinear interaction with quasi-2D subharmonic merging instabilities.

Recent work on pipe flow (Pringle \& Kerswell 2010; Pringle, Willis \& Kerswell 2012) and plane Couette flow (Monokrousos et al. 2011; Rabin, Caulfield \& Kerswell 2012) has demonstrated the utility of the (natural) nonlinear generalization of the direct-adjoint looping used here to consider the identification of so-called 'minimal seeds' of turbulence, i.e. the perturbations with the smallest initial energy which can lead to triggering turbulent transition. It is an interesting open question as to whether such inherently nonlinear optimization calculations can identify a connection between the H-type perturbations (for example) discussed here and the nonlinear mechanisms which actually trigger turbulence in time-evolving mixing layers at sufficiently high Reynolds numbers.

\section{Acknowledgements}

Preliminary calculations on this problem were conducted by S. Iams for her M.Phil. at the BP Institute, University of Cambridge. The adjoint equations and the optimization algorithm were implemented in the version of the DNS code developed by A. Deloncle. The Krylov method routine was adapted in a pre-existing version by S. Ortiz. This manuscript has been substantially improved by the consideration of the insightful and constructive comments of Professor W. R. Peltier, and two anonymous referees. C.A. acknowledges financial support for his $\mathrm{PhD}$ from the Chilean and French governments. The research activity of C.P.C. is supported by EPSRC Research Grant EP/H050310/1 ‘AIM (Advanced Instability Methods) for industry'. C.P.C. would also like to acknowledge the generous hospitality of the Hydrodynamics Laboratory (LadHyX) École Polytechnique/CNRS during the production of this manuscript.

\section{REFERENCES}

Antkowiak, A. \& BRANCHeR, P. 2007 On vortex rings around vortices: an optimal mechanism. J. Fluid Mech. 578, 295-304.

BASAK, S. \& SARKAR, S. 2006 Dynamics of a stratified shear layer with horizontal shear. J. Fluid Mech. 568, 19-54.

BAYLY, B. J. 1986 Three-dimensional instability of elliptical flow. Phys. Rev. Lett. 57 (17), 2160-2163.

Brown, G. L. \& RoshKo, A. 1974 On density effects and large structure in turbulent mixing layers. J. Fluid Mech. 64, 775-816.

Butler, K. M. \& FARRell, B. F. 1992 Three-dimensional optimal perturbations in viscous shear flow. Phys. Fluids A 4, 1637-1650.

Caulfield, C. P. \& Kerswell, R. R. 2000 The nonlinear development of three-dimensional disturbances at hyperbolic stagnation points: a model of the braid region in mixing layers. Phys. Fluids 12, 1032-1043.

Caulfield, C. P. \& Peltier, W. R. 2000 The anatomy of the mixing transition in homogeneous and stratified free shear layers. J. Fluid Mech. 413, 1-47. 
Corcos, G. M. \& LiN, S. J. 1984 The mixing layer: deterministic models of a turbulent flow. Part 2. The origin of the three-dimensional motion. J. Fluid Mech. 139, 67-95.

Corcos, G. M. \& Sherman, F. S. 1984 The mixing layer: deterministic models of a turbulent flow. Part 1. Introduction and the two-dimensional flow. J. Fluid Mech. 139, 29-65.

Dimotakis, P. E. 2005 Turbulent mixing. Annu. Rev. Fluid Mech. 37, 329-356.

Donnadieu, C., Ortiz, S., Chomaz, J.-M. \& Billant, P. 2009 Three-dimensional instabilities and transient growth of a counter-rotating vortex pair. Phys. Fluids 21 (9), 094102.

Drazin, P. G. \& REID, W. H. 1981 Hydrodynamic Stability. Cambridge University Press.

Ellingsen, T. \& Palm, E. 1975 Stability of linear flow. Phys. Fluids 18, 487-488.

FARRELl, B. F. \& IOANNOU, P. J. 1993a Optimal excitation of three-dimensional perturbations in viscous constant shear flow. Phys. Fluids A 5, 1390-1400.

FARrell, B. F. \& IOANnOU, P. J. $1993 b$ Perturbation growth in shear flow exhibits universality. Phys. Fluids A 5, 2298-2300.

FARrell, B. F. \& IOANnOU, P. J. 1996 Generalized stability theory. Part II: nonautonomous operators. J. Atmos. Sci 53, 2041-2053.

Guegan, A., Huerre, P. \& Schmid, P. J. 2007 Optimal disturbances in swept Hiemenz flow. J. Fluid Mech. 578, 223-232.

HAZEL, P. 1972 Numerical studies of the stability of inviscid stratified shear flows. J. Fluid Mech. 51, 39-61.

HILL, D. C. 1995 Adjoint systems and their role in the receptivity problem for boundary layers. J. Fluid Mech. 292, 183-204.

Hussain, A. K. M. F. 1983 Turbulence and Chaotic Phenomena in Fluids (ed. T. Tatsumi), pp. 453-460. North-Holland.

Kerswell, R. R. 2002 Elliptical instability. Annu. Rev. Fluid Mech. 34, 83-113.

KlaAssen, G. P. \& Peltier, W. R. 1985 The onset of turbulence in finite-amplitude Kelvin-Helmholtz billows. J. Fluid Mech. 155, 1-35.

Klafissen, G. P. \& Peltier, W. R. 1989 The role of transverse secondary instabilities in the evolution of free shear layers. J. Fluid Mech. 202, 367-402.

Klafissen, G. P. \& Peltier, W. R. 1991 The influence of stratification on secondary instability in free shear layers. J. Fluid Mech. 227, 71-106.

LANDAHL, M. T. 1980 A note on an algebraic instability of inviscid parallel shear flows. $J$. Fluid Mech. 98, 243-251.

LASherAs, J. C. \& CHOI, H. 1988 Three-dimensional instability of a plane free shear layer: an experimental study of the formation and evolution of streamwise vortices. J. Fluid Mech. 189, 53-86.

Metcalfe, R. W., Orszag, S. A., Brachet, M. E., Menon, S. \& Riley, J. J. 1987 Secondary instability of a temporally growing mixing layer. J. Fluid Mech. 184, 207-243.

Monokrousos, A., Bottaro, A., Brandt, L., Di Vita, A. \& Henningson, D. S. 2011 Nonequilibrium thermodynamics and the optimal path to turbulence in shear flows. Phys. Rev. Lett. 106, 134502.

ORR, W. M'F. 1907 The stability or instability of the steady motions of a perfect liquid and of a viscous liquid. Part I: a perfect liquid. Proc. R. Irish Acad. A 27, 9-68.

OrTIZ, S. \& ChOMAZ, J.-M. 2011 Transient growth of secondary instabilities in parallel wakes: anti lift-up mechanism and hyperbolic instability. Phys. Fluids 23 (11), 114106.

Pierrehumbert, R. T. \& Widnall, S. E. 1982 The two- and three-dimensional instabilities of a spatially periodic shear layer. J. Fluid Mech. 114, 59-82.

Potylitsin, P. G. \& PeltieR, W. R. 1998 Stratification effects on the stability of columnar vortices on the f-plane. J. Fluid Mech. 355, 45-79.

Pringle, C. C. T. \& Kerswell, R. R. 2010 Using nonlinear transient growth to construct the minimal seed for shear flow turbulence. Phys. Rev. Lett. 105, 154502.

Pringle, C. C. T., Willis, A. P. \& Kerswell, R. R. 2012 Minimal seeds for shear flow turbulence: using nonlinear transient growth to touch the edge of chaos. J. Fluid Mech. 703, 415-443.

Rabin, S. M. E., Caulfield, C. P. \& Kerswell, R. R. 2012 Triggering turbulence efficiently in plane Couette flow. J. Fluid Mech. 712, 244-272. 
Rogers, M. M. \& Moser, R. D. 1992 The three-dimensional evolution of a plane mixing layer: the Kelvin-Helmholtz rollup. J. Fluid Mech. 243, 183-226.

Schmid, P. J. 2007 Nonmodal stability theory. Annu. Rev. Fluid Mech. 39, 129-162.

Schoppa, W., Hussain, F. \& Metcalfe, R. W. 1995 A new mechanism of small-scale transition in a plane mixing layer: core dynamics of spanwise vortices. J. Fluid Mech. 298, 23-80.

SMyth, W. D. \& Peltier, W. R. 1994 Three-dimensionalization of barotropic vortices on the f-plane. J. Fluid Mech. 265, 25-64.

SQuire, H. B. 1933 On the stability of three-dimensional disturbances of viscous flow between parallel walls. Proc. R. Soc. Lond. A 142, 621-628.

Vitoshinin, H., Heifetz, E., GelfGat, A. Yu. \& Harnik, N. 2012 On the role of vortex stretching in energy optimal growth of three-dimensional perturbations on plane parallel shear flows. J. Fluid Mech. 707, 369-380.

WAlefFe, F. 1990 On the three-dimensional instability of strained vortices. Phys. Fluids A 2, 76-80.

WinANT, C. D. \& BRowAND, F. K. 1974 Vortex pairing: the mechanism of turbulent mixing-layer growth at moderate Reynolds number. J. Fluid Mech. 63, 237-255. 